Robotica: (2021) volume 39, pp. 1368-1389. (C) The Author(s), 2021. Published by Cambridge University Press. This is an Open Access article, distributed under the terms of the Creative Commons Attribution-NonCommercial-NoDerivatives licence (http://creativecommons.org/licenses/by-nc-nd/4.0), which permits non-commercial re-use, distribution, and reproduction in any medium, provided that no alterations are made and the original article is properly cited. The written permission of Cambridge University Press must be obtained prior to any commercial use and/or adaptation of the article. doi:10.1017/S0263574720001228

\title{
Variable Inverted Pendulum Applied to Humanoid Motion Design
}

\author{
Teresa Zielinska†*๑, Gabriel R. Rivera Coba $\ddagger$ \\ and Weimin Ge $q^{*}$
}

$\dagger$ Faculty of Power and Aeronautical Engineering, Warsaw University of Technology, Warsaw, Poland $\ddagger$ Ecuador, Quito, sector: La Concepción de Alpahuma, Alangasí, Calle A, Betania, ref. Urbanización Miradordel Colegio.E-mail: grivera@soindec.com

ФSchool of Mechanical Engineering, Tianjin University of Technology, Tianjin, China

(Accepted November 27, 2020. First published online: February 4, 2021)

\begin{abstract}
SUMMARY
Double inverted pendulum model, stationary or on a cart, is computationally the simplest out of the range of reasonable models used for anthropomorphic robots motion synthesis. However, it is still not sufficient for describing more complex situations. The novel concept of variable double inverted pendulum (VDIP) for static postures and VDIP on cart (VDIPC) for dynamic cases is proposed. It provides a simplified but a sufficiently accurate tool for planning the human-like static and dynamic robot postures. Its variable parameters enable the description of both human static postures and motion dynamics. The variable length of the lower link is essential for the representation of postures attained by bending legs. The studies of a set of static and dynamic postures were used for deducing and verifying the locations of lower and upper joint of a double pendulum and the point masses. To justify the concept, human body and pendulum behaviors are compared taking into account a typical model of the human body. Static analysis was conducted by considering static human postures. Dynamic conditions were analyzed using the data acquired from human motion and thus the VDIPC definition was established. The zero moment point trajectories of the human and of VDIPC were compared, validating the correctness of VDIPC in dynamic situations. The formal description of VDIPC is provided together with the torques equilibrium condition needed for evaluating the dynamic postural stability, with the VDPIC representing the robot configuration. The VDPIC state equations are formulated in a form required by the predictive control method. The paper contributes to the motion synthesis methods of anthropomorphic robots taking into account postural control.
\end{abstract}

KEYWORDS: Double inverted pendulums; Humanoids; Motion synthesis; Human postures and movements.

\section{Introduction}

For robots acting on a human-oriented environment and interacting with a human, it is important not only to walk and manipulate but also to adjust the whole body posture in a human-like way. The advantages of having a human-like robot are numerous, but especially a typical environment does not have to be modified, and robots resembling humans can do the daily chores using tools designed for humans. A human-like robot can work as a care provider without disquieting the patients by its appearance or by using unusual equipment. According to Japanese Robot Strategy presented by Walters, ${ }^{38}$ humanoids are expected mainly to take care of the elderly.

*Corresponding authors. E-mail: teresaz@meil.pw.edu.pl, geweimin@email.tjut.edu.cn 
Many different methods are used for humanoid motion synthesis. In the simplest approach, the captured human motion data are used as an input for the robot to follow. Unfortunately, in this case motion adaptation to varying external conditions is not possible. The alternative analytical methods generating the humanoid postures (e.g., the methods using the augmented Jacobian) are computationally demanding, and they are mainly limited to the imposed constraints. It is also not guaranteed that the obtained posture will be always human-like; therefore, additional conditions must be introduced (e.g., Shi Zhong et al. ${ }^{30}$ ). Moreover, with complex formalism and lack of prediction the real-time implementation is here problematic, as the humanoid must react almost immediately to the changes in the environment in a human-like way. Even for a typical gait of the robot, several factors must be considered simultaneously: the leg-end trajectories, foot placement strategy, and a plan of upper body motions to assure the postural stability. A motion planner which is able to deal with all those aspects simultaneously is still sought for.

Over 40 years ago, Vukobratovic ${ }^{35}$ defined the zero moment point (ZMP) criterion, commonly used for humanoid motion synthesis. In the later works by his research group (Vukobratovic et al ${ }^{36,37}$ ), a humanoid was described as a multi-body system and ZMP criterion was supporting the motion design. The papers by Hirai et al., ${ }^{13}$ Wang et al., ${ }^{39}$ and Yamaguchi et al. ${ }^{42}$ are examples of research where quite detailed whole body model was used for the purpose of motion generation. Similarly, Harada et al. ${ }^{10}$ considered a whole body model, while dealing with obstacles in this case. The body parameters such as geometry, mass, inertia, and the location of each link center of mass $(\mathrm{CoM})$ influence motion dynamics. Unfortunately, detailed models produce computationally intensive solutions.

Berenson et al. ${ }^{4}$ proposed a universal motion planning strategy using the so-called task space regions. It is a probabilistic, sample-based approach that nominally can avoid the heavy computational load. Three main components of the method are constraint manifold, constraint-satisfaction strategy, and planning algorithm called constrained bi-directional rapidly exploring random tree. The constraint manifold contains all configurations that do not violate the imposed constraints. Unfortunately, this method has some limitations concerning motion generation for load lifting tasks. The method conducts a search in configuration space for configurations producing a trajectory resulting in a motion passing through the required footsteps; however, postural stability conditions are not taken into account. Therefore, this approach has limitations concerning motion generation, where such dynamical effects as those appearing in load lifting tasks are relevant. In the paper by Qiu et al., ${ }^{29}$ the robot body motion is described by the trajectory of its CoM and predefined footholds. The balance constraints are formulated in the form of permissible friction cone of the foothold. The trajectory of the mass center is represented by a B-spline function with parameters selected through an optimization process requiring that jerk and motion duration be minimized, satisfying the balance constraints. The force moments equilibrium condition is not considered. Such an approach does not specify how to define the trajectory of the CoM when the robot has to adjust a stable posture keeping constant footholds or how to design the motion when gaining dynamic balance. A more complex approach was applied by Ott et al., ${ }^{27}$ where the task was to design a postural balance controller by adjusting the contact forces. The robot was described as a closed mechanical chain (closure through the ground). The dynamic equilibrium was analyzed using the multi-body approach taking into account the distribution of the net wrench (forces and torques) so that the posture and mass position produce the required contact conditions. The force constraints were formulated in terms of friction cones, taking into account the ZMP requirements.

Other approaches use biological inspiration. Often models of central pattern generator (CPG) are utilized. In opposition to modeling dynamics, which is a purely theoretical approach, the CPG approach refers to a biological template. CPG is a neural structure located in the spinal cord. Among others, it generates the rhythm of locomotion. Locomotion of many animals (including human beings) is governed by CPG (Ijspeert ${ }^{16}$ ). The regular walking pattern is generated unconsciously without involvement of high-level control (brain). In the article by Hase et al. ${ }^{11}$ the gait generator with sensory feedback was presented, together with the model of the musculoskeletal system. Up to 50 walking steps had been generated successfully. The system behaved well also in the presence of disturbances. A CPG-based gait was presented by Hyon et al. ${ }^{15}$ for a human-size robot, confirming that by using this approach balancing and walking on uneven terrain can be achieved. Despite its success, the CPG-based approach is mainly tailored to a specific range of movements and to a specific robot. Moreover, the number of unknown parameters is usually high and it is difficult to determine their values. 
In the paper by Zielinska, ${ }^{43}$ trajectories of recorded human gait were used to facilitate the search for appropriate parameter values. The CPG was described by the Van der Poll coupled oscillators with 24 unknown parameters. Once appropriate parameters were selected the oscillator, equations produced leg joint angle trajectories for a typical human gait. The strategy for parameter modification resulting in gait transitions was investigated. The real-time-based gait generator using the modified Van der Poll formula with sensory feedback was proposed. To a certain extent, disturbance rejection was achieved. The method was tested using a 12 DOF (Degrees of Freedom) biped (Zielinska et al. ${ }^{44}$ ).

Real-time-based motion generation methods often use preview control utilizing state equations of an inverted pendulum. The body is reduced to a single point mass on a massless rod. The paper Kajita et al. ${ }^{17}$ proposes a simple concept of gait generation using a 3D linear inverted pendulum (LIP). The primary objective of the model preview control is to obtain the pendulum CoM motion trajectory resembling that of a human CoM trajectory. Using the simplified model of a humanoid, the predictive controller generates the trajectory of the CoM. The control system of the real robot tracks it the best it can. A model of an inverted pendulum on a cart moving on a table was proposed as a model of human motion (Kajita et al. ${ }^{19}$ ). The trajectory of the ZMP was provided, and the CoM motion, assuring close tracking of the predefined ZMP trajectory, was obtained using the preview control method.

The paper by Xue et al. ${ }^{41}$ presents an inverted pendulum model combined with the models of flexible joints achieving fast, real-time motion generation. Lanari et al. ${ }^{21}$ studied LIP dynamics to identify stable and unstable walking states. They obtained the so-called Bounded Solution for avoiding the unstable states. Unfortunately, the LIP model is not able to represent the behavior of the upper part of the body. Therefore, double inverted pendulum (DIP) models were used, for example, Hwang et al. ${ }^{14}$ presented human-like balancing motion using DIP. There two actions performed by a human were analyzed. The human motion capture data were used to develop a hip balancing strategy providing human-like postural adjustments while keeping the ZMP inside the support polygon.

The spring loaded inverted pendulum (SLIP) model was used to study fast locomotion of humans and animals having legs. Such a model represents well the dynamic behavior of the CoM during running (Full et al. ${ }^{8}$ ). SLIP models are also used in dynamic controllers of hopping robots, for example, Mordatch et al. ${ }^{23}$ applied the SLIP model and a simple planner generating walking, running, and jumping motions of bipeds. The model was successfully exploited simulating the locomotion on uneven terrain. The single or double inverted pendulum models are also commonly used to study human balancing (Alexandrov et al., ${ }^{1}$ Kasaei Mohammadreza ${ }^{20}$ ). They enable human-like postural recovery when the normal motion pattern is disturbed. In Alexandrov et al. and Lippi et al., ${ }^{1,22}$ the mechanism of human balance was investigated using the LIP model. Zielinska et al. ${ }^{45}$ employed a disturbance rejection controller for postural balancing taking into account the standard double pendulum model.

The analysis of the state of the art indicates that LIP- or DIP-based, simple models are mainly used for two purposes. The first one is to study human balance strategies. The second purpose is to apply those models to the design of the reference trajectories of ZMP or the trajectories of the robot CoM. Both are used in computationally efficient model preview controllers. In the first case, simple LIP or DIP models are sufficient; however, this is not so in the second case. In this case, the models should enable the design of the motion trajectories of the main point masses, reflecting the standing, walking, or running postures, but also while performing more complex tasks, including transfer of objects and reaction to dynamical disturbances affecting displacements. The above observation underlies the motivation of the presented work. The objective is to find a simplified model of a human body, which provides sufficient information about its posture and motion, to facilitate the design of motion of humanoids.

The paper is organized as follows. Inverted pendulum models are summarized in Section 2 and proposed model is introduced. In Section 3, the data collection method is summarized. Section 4 describes static postures of the human body using the modified inverted double pendulum (VDIP model). The positions of the lower and upper joints are defined referring to the structure of a human body. In Section 5, the dynamic analysis is performed considering two cases stepping forward and backwards, and push recovery. For both cases, the ZMP and CoM trajectories for VDIPC and a more detailed model of the human body are compared. The discussion of results together with the formal models is given in Section 6. The paper ends with conclusions. The overall work makes a step towards 
modeling human static and dynamic postures using simplified models suitable for investigations of postural stability and for real-time generation of motion templates. This work reports our research progress towards simplified models describing the human body. Detailed discussion of the obtained results can be found in Coba. ${ }^{6}$

\section{Inverted Pendulum Models}

\subsection{Existing approaches}

As it was already mentioned, the simplified models are computationally efficient and are suitable for real-time implementation in model preview control methods (e.g., Audren Herve et al. ${ }^{2}$ ) or in model predictive methods (e.g., Scianca et al. ${ }^{31}$ ), for generating the motion trajectories. Thus, the inverted pendulum-based methods are very suitable for real-time applications.

The LIP model is mainly used for describing the single support phase of a gait (Chengju Liu, ${ }^{5}$ Kajita et al., ${ }^{17}$ Xue et $a .^{41}$ ). The lower joint of the pendulum (pendulum pivot point), in such a situation, is located at the ankle of the humanoid robot, or at a point on the ground below the ankle, or even below the ground for more accurate representation of walking (Omran et al. ${ }^{26}$ ). It can also overlap with the ZMP (Kajita et al. ${ }^{18}$ ). As it has been already mentioned, due to the reduction of the body to a single point mass attached to a weightless rod, the information about the posture, when using the LIP model, is completely lost. For example, if the length of the link is fixed, the posture of a crouching humanoid cannot be described.

The positions of the lower (base) and the upper joint of a double pendulum are crucial to modeling of human body postures and locomotion. The literature indicates how to choose the location of the lower joint for normal walking (Hayot et al. ${ }^{12}$ ); however, other modes of locomotion have not been investigated thoroughly.

Zurawska et al. ${ }^{46}$ used a DIP with moving masses to study the motion coordination of upper and lower parts of the human body. The human motion capture data for four different movements were analyzed, and a strong coincidence with DIP mass displacement was noticed. A double pendulum with moving masses was also used by Villalobos et al. ${ }^{34}$ to analyze the postural recovery after unexpected push. The DIP model consists of two links and two point masses corresponding to the upper and lower part of the body; therefore, the whole posture can be better reflected. Unfortunately, such model has still limitation; it can describe only the range of standing postures when the legs are not bent in the knees. This limits its usefulness for modeling the humanoid postures.

Beside single and double inverted pendulums, a triple inverted pendulum can also be considered for modeling the human body; however, the partitioning of the body in this case is not defined clearly. For the DIP model, the division of the human body into two parts is obvious - the division line is located at the level of hips or waist. The three segment models of the human body, consisting of thigh, shank, and upper part of the body, considered in the literature, are usually not treated as inverted pendulums, but as kinematic chains (e.g., Asker et al. ${ }^{3}$ ). It must be noted that positions of the thigh and shank are determined by the leg-end trajectory and not by the dynamical equilibrium requirement. Therefore, not the thigh and shank positions separately, but the position of the point masses of the lower and upper body parts are relevant to dynamical balancing.

In summary, in relation to LIP, the classic DIP models reflect better the human body structure; however, they still have some limitations. Treating the previous research as a foundation, the current work introduces an enhanced version of an inverted pendulum, enabling the consideration of more complex postures and non-periodic movements.

\subsection{Proposed model}

As it was mentioned above, the currently used DIP model with fixed length of the links and fixed position of the masses has serious limitations. The left-hand side of Fig. 1 illustrates the weakness of DIP with fixed-parameter links. Usually the lower joint of the pendulum is located in the ankle. The lengths of the lower and upper link correspond to the distances assumed for the standing posture, from the ankle to the waist and from the waist to the top of the head. It is easy to notice that a pendulum with a fixed structure cannot reflect properly those postures for which the legs are bent in the knees. Setting the location of the point masses of the upper and lower link in the same places as for a standing human and preserving a constant length of the lower link, a very strange pendulum 

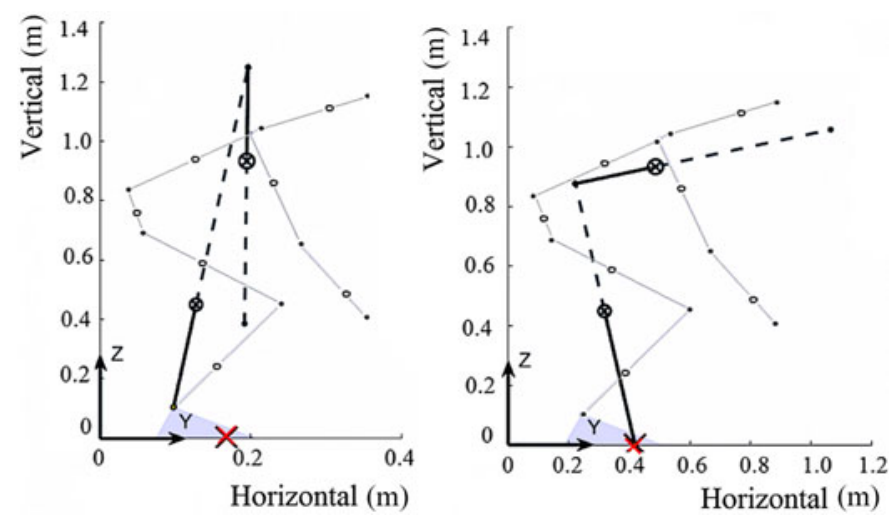

Fig. 1. Crouching posture. This posture cannot be represented adequately using fixed-parameter DIP (left), but VDIPC reflects this posture correctly (right).

posture is obtained, which is far apart from that of the human. The upper link hangs down and the tip of the pendulum is far from the location of the human head, as the left part of Fig. 1 illustrates.

Noticing such shortcoming and keeping in mind that inverted pendulum-based methods are very suitable for real-time applications, we studied how such model can be modified describing more precisely the human postures and dynamical movements. By analyzing examples of static and dynamic human postures, it was deduced and verified the locations of lower and upper joints of a double pendulum, proposing the variable double inverted pendulum (VDIP) for static postures and VDIP on a cart (VDIPC) for dynamic cases. This concept extends the DIP model presented by Hwang et al.. ${ }^{14}$

The pendulum is characterized by moving masses and variable link lengths. The lower link length corresponds to the current distance from the lower joint (located at the projection of the overall body CoM onto the ground) to the waist. The length of the upper link remains constant and is equal to the distance from the waist to the tip of the head, measured when the torso and the neck are straightened up. Figure 1 illustrates that the posture which cannot be represented accurately by DIP is reflected correctly using the VDIPC model.

\section{Data Collection and Preprocessing}

The presented research takes into account human postures and motion data. The below analyzed static data were collected for a 24-year-old healthy female subject, $151 \mathrm{~cm}$ tall, weighing $45 \mathrm{~kg}$. The dynamic data were collected for a 24-year-old healthy female, $168 \mathrm{~cm}$ tall, weighing $54 \mathrm{~kg}$. The normality of motion behaviors of the person taking part in the dynamical analysis was verified by the Motion Laboratory of a hospital.

The necessary anthropomorphic data were obtained taking into account that the relation between the weight of the body segments and the location of their CoM depends on the total body weight, as defined by Winter. ${ }^{40}$ Other anthropometric data were extracted from Contini, ${ }^{7}$ including the relationship between segment lengths with respect to the total body height. An anthropometric survey conducted by Gordon et al., ${ }^{9}$ providing a significant amount of data, was also used in our investigations. Finally, the data describing the body parameters were calculated taking into account the following information: gender, height, and mass.

The static postures were recorded using a typical still camera. The image distortion was removed by filtering. The dynamical movements were recorded using a professional motion recording system VICON. The data were processed using the assisting software presenting clear joint trajectories. Such a set of data was sufficient to build the models of the human body and the inverted pendulum used in this work. Each recording lasted from the start to the end of the subject's motion, with an additional time margin. The motion was executed with a speed comfortable for the examined person. In the cases where time was a relevant factor (dynamic analysis), the exact time is defined in the farther descriptions.

The captured data were filtered to reject the noise and supplemented to produce the missing data using the software provided by VICON. The data were represented numerically. The open access 3D Motion Kinematic \& Kinetic Analyzer (Mokka) software was used to visualize the positions of markers. 


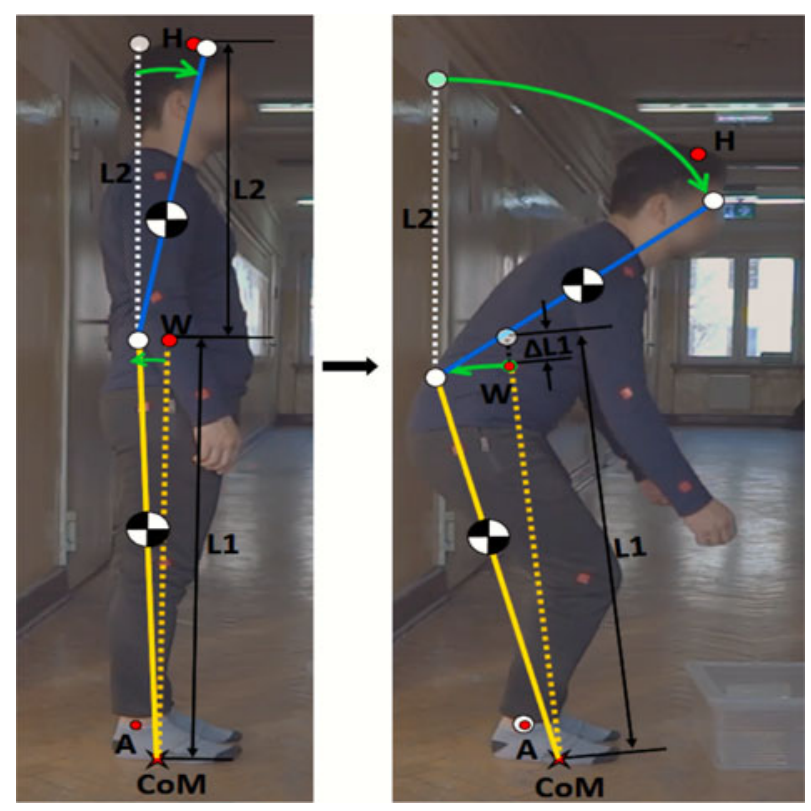

Fig. 2. Human posture modeled by the VDIP. In the figure, CoM denotes the CoM projection onto the ground.

\section{Definition of VDIP: Static Analysis}

Classical double pendulum with fixed link lengths and the lower joint located in the ankle is not sufficient to represent many human postures, as it has been already mentioned and illustrated in the left part of Fig. 1. The right part of this figure shows that the double pendulum with variable length of the lower link and the lower joint located in the projection of CoM onto the ground represents human posture more accurately. The aim is the definition and validation of our double pendulum model. For this purpose, a set of static human postures (of males and females) executing a wide range of activities was analyzed.

VDIP models describing each posture were produced. Figure 2 illustrates the method of VDIP parameter adjustment. A, W, and $\mathrm{H}$ denote the points marking the ankle, waist, and head of the human subject, respectively. $L 1$ is the lower link length, which is a variable quantity. The location of the lower link is defined by two points: the projection of the overall body CoM onto the ground and the position of the CoM of the lower body part. The link is extended to the waist height. It should be noted that the waist height is measured from the ground to the horizontal line passing through the waist. The upper link length $L 2$ is fixed and is equal to the vertical distance from the waist height to the tip of the head when the torso and the neck are straightened up. The upper link position is defined by the end point of the lower link and the position of the CoM of the upper part (Fig. 2).

The position of the CoM of any item of interest (whole body, body segment, or link) is obtained using the following general formula:

$$
{ }^{k} s_{c}=\frac{\sum_{i=1}^{n}{ }^{k} s_{i} \cdot m_{i}}{\sum_{i=1}^{n} m_{i}}
$$

where $s \in\{x, y, z\}$ and $x, y, z$ are the respective coordinates of point masses of segments composing the considered human subject or VDIP (item of interest), where $n$ is the number of these segments. The leading superscript $k$ designates the data set for a specific static posture or an image frame in the sequence of recorded frames for dynamic movements. $m_{i}$ is the mass of the $i$-th segment (in kilograms). When calculating the position of the overall CoM of the double pendulum, two point masses of DIP are taken into account, that is, $n=2$. In the case of a human body, the CoM position is obtained taking into account eleven segments, that is, $n=11$. When evaluating the position of the CoM of the upper link of DIP, we take into account six segments of the human upper body, and when evaluating the position of the CoM of the lower link five segments of the lower part of the human body are considered. The positions of the human body partial masses are derived from anthropometric data. 

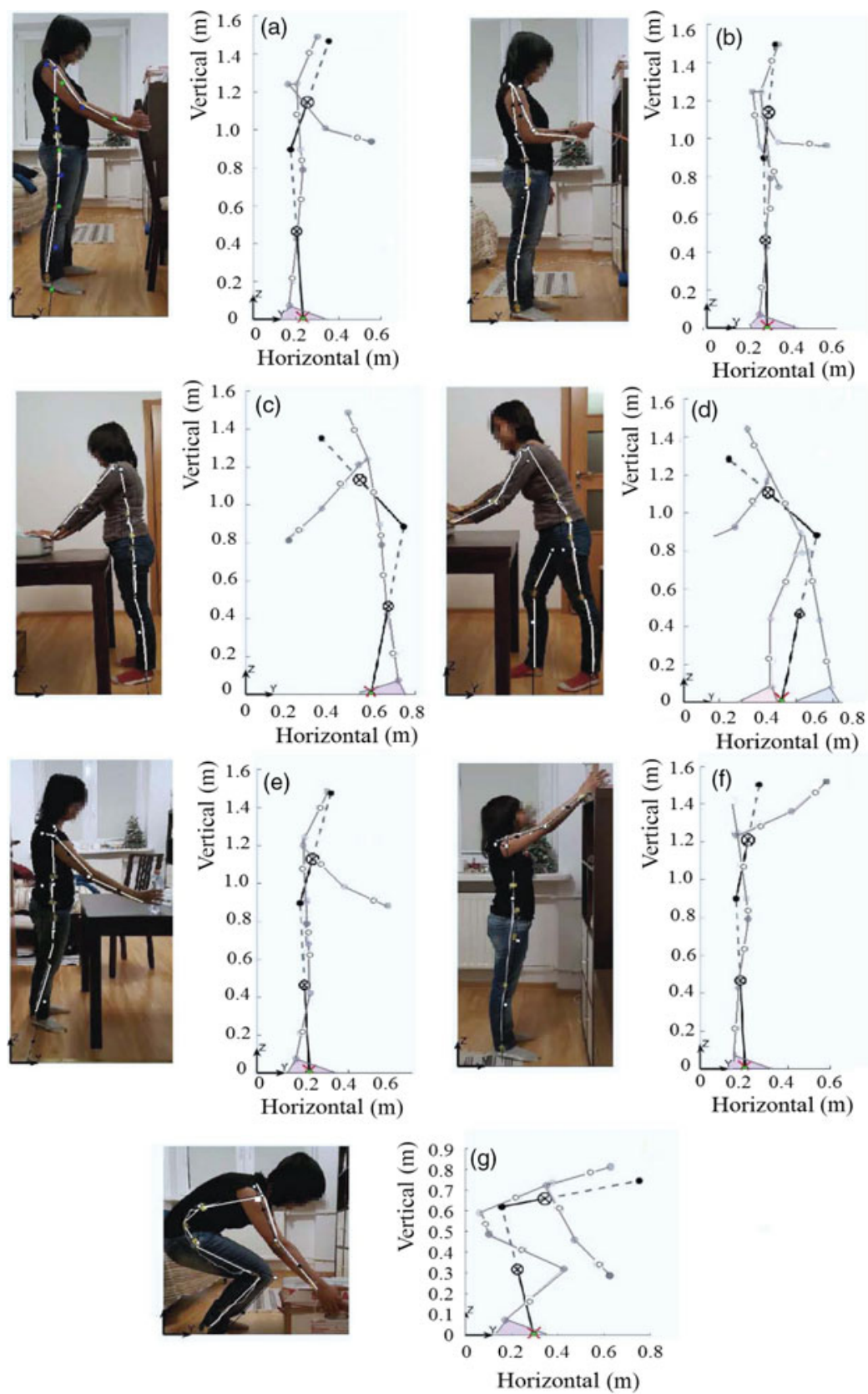

Fig. 3. Pulling an object (a, b), pushing an object (c, d), and picking an object (e, f, g).

The configurations of a DIP were defined by considering the location of human body member masses, projection of the total CoM onto the ground, and the waist height. It was assumed that the human body keeps its orientation without sideways inclination. Figure 3 illustrates selected examples using a planar representation; however, the lower joint pivot point position was obtained in 3D space. The positions are symmetrical with respect to the sagittal plane. This means that the postural stability in the sagittal plane is affected, but in the frontal plane it is not. For the calculations, a 3D model of the human body was used. Each point mass was described by $x, y, z$ coordinates. Due to symmetry, the projection of the total mass (lower joint pivot point) marked by red cross is located in the sagittal plane (between the footprints). The photographs show: (a) pulling an object using two hands, (b) pulling a light object with one hand, (c) pushing a light object using two hands, (d) pushing a heavy object using two hands, (e) picking an object at waist height with one hand, (f) picking an object at above the head height using two hands, and (g) picking an object from the floor using two hands. On the left-hand side, the person's photographs are shown together with the line segments representing 
(a)

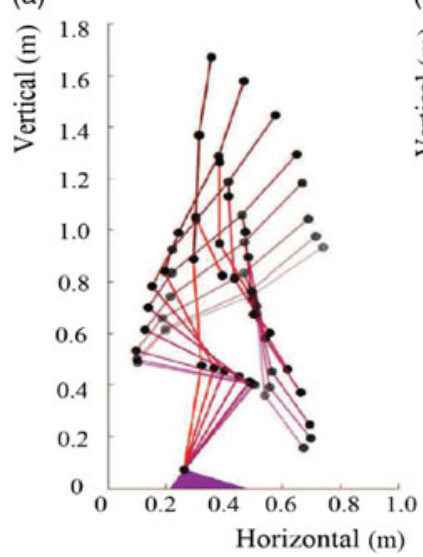

(b)

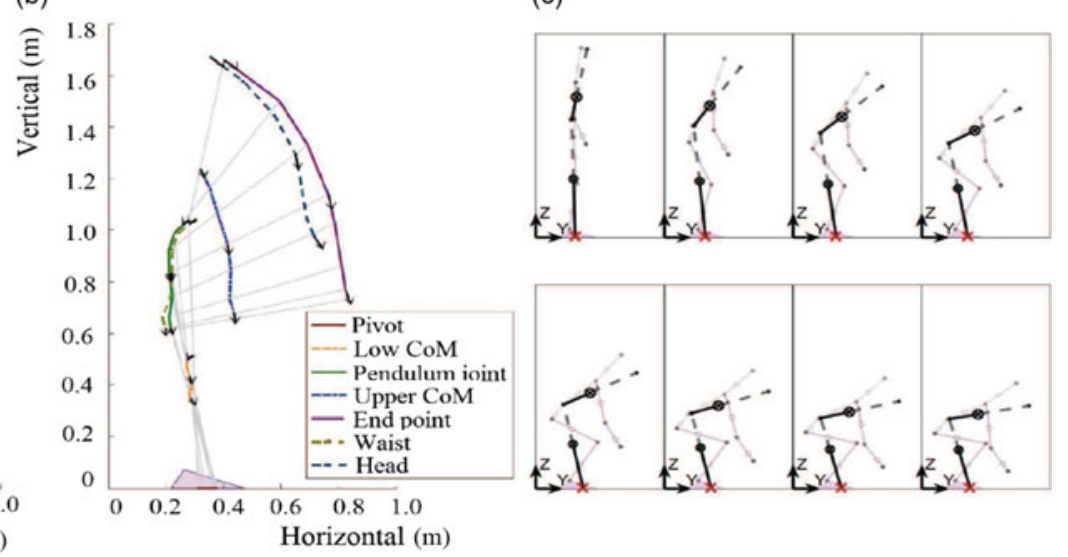

Fig. 4. Transition from standing to crouching position (a), VDIP configuration with relevant trajectories (b), and selection of image frames (c).

the posture. On the right-hand side, the VDIPC is drawn (darker lines) using stick diagram as the background (lighter color). The localization of the point masses of the human body segments is indicated (light circles) together with the point masses of the pendulum (crossed dark circles). The localization of body partial masses was obtained using the anthropometric data. The position of the total CoM was evaluated, its projection (marked by red cross) indicates the pendulum lower joint pivot point.

To better illustrate that the postural transition is well represented by VDIP, the change from a standing to a crouching posture is shown in Fig. 4. Figure 4(a) presents the sick diagrams with the relevant points. Figure 4(b) shows the trajectories of the relevant points together with the links of the pendulum and the positions of their CoMs. Figure 4(c) illustrates how the VDIP adjusts to the postural changes.

\section{Dynamics Analysis}

Several dynamic activities were studied. Out of those we discuss three representative cases: the forward-backward stepping and postural stability recovery after unexpected push from the back and the side. Such selection enables the study of slow dynamics (stepping) versus fast dynamics (push recovery). For all cases, we compared the human motion trajectories of relevant body points. Next we compared the ZMP trajectories for humans and VDIPC. Finally, we compared human postures with their VDIPC representations.

\subsection{Data processing}

The data captured by the VICON system were filtered to reject the noise and supplemented to produce the missing data using the software provided by VICON. The data were represented numerically. The open access 3D Motion Kinematic \& Kinetic Analyzer (Mokka) software was applied to visualize the positions of markers, which was relevant to our studies. The data processed by VICON assisting software produced the human body joint trajectories. Those trajectories, together with the anthropometric data, are sufficient to build and animate the human body models and to investigate the proposed inverted pendulum.

\subsection{Stick diagram of the human body and pendulum motion}

A stick diagram of the body was constructed out of 11 segments: (1) head and neck, (2) trunk, (3) pelvis, (4) left upper arm, (5) left forearm, (6) right upper arm, (7) right forearm, (8) left thigh, (9) left shank and foot, (10) right thigh, and (11) right shank and foot, taking into account the positions of markers. More than 30 markers were used, but 22 of them were selected as a sufficient set for building the stick diagrams and for motion analysis. Anthropometric data extracted from the works by Contini, ${ }^{7}$ Gordon et al., ${ }^{9}$ and Winter ${ }^{40}$ were also used, supplementing the information provided by the markers. These data describe the values and locations of partial masses. The resultant stick diagram is shown in Fig. 5. In Fig. 5(a), the segmented model of the human body is shown with point 
(a)

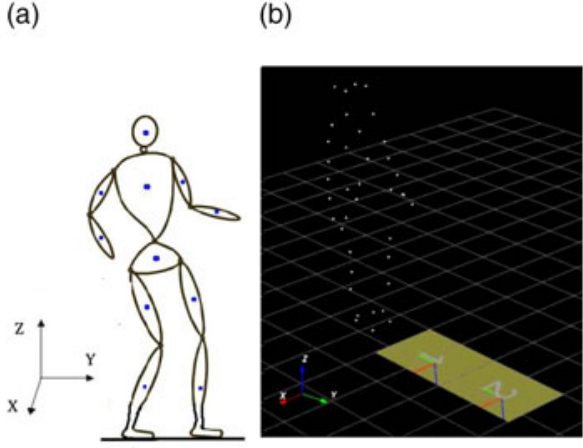

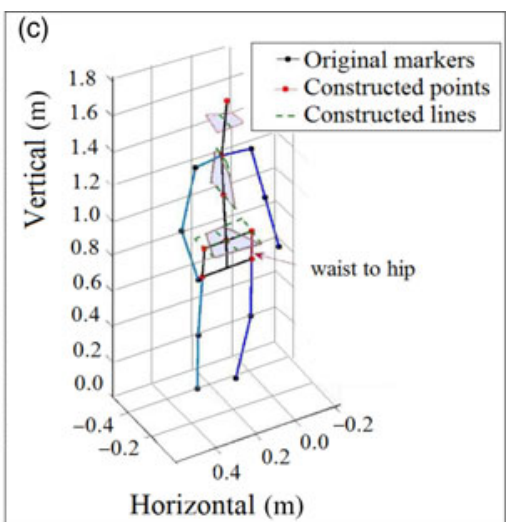

Fig. 5. The segmented model of the human body (a), view of the markers presented by the Mokka software (b), and reconstructed stick diagram (c).
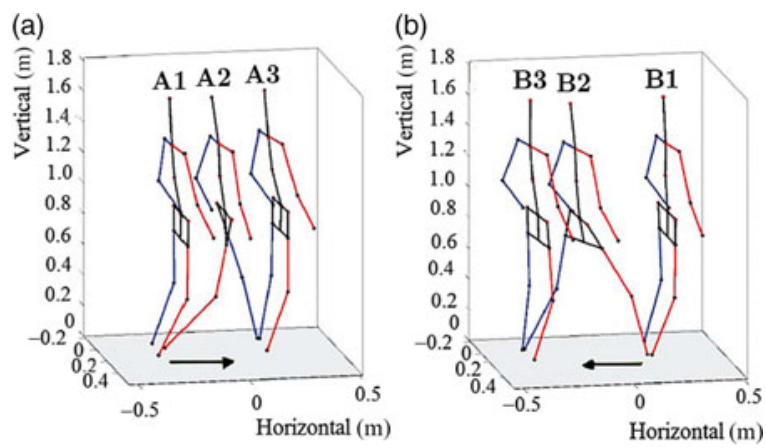

Fig. 6. Changes of the body posture, represented by a stick diagram, in the case of making a step forward (a) and making a step to the back (b).

masses marked by blue dots; the stick diagram reconstructed using the markers is shown in Fig. 5(b) with some relevant points (black dots point out joint positions, red dots show some relevant points, the positions of which were reconstructed using the positions of markers and anthropometric data; additionally some construction lines are shown which we used to construct the 3D representation). Once the stick diagram was obtained and the point masses of each segment were located, it was possible to establish the model of a double inverted pendulum on a cart (VDIPC). The pelvis, thighs, and shanks with feet contribute to the lower point mass of VDIPC, while the masses of remaining segments contribute to the upper mass.

\subsection{Stepping: active motion}

This section compares the human and VDIPC behavior for the case of a human stepping forward and backwards from a stand-still posture (double support). The following events are distinguished (Fig. 6):

- A1 $-0.80 \mathrm{~s}$ : the right leg single support phase starts at $0.80 \mathrm{~s}$ from the beginning of the recording; the left leg starts its transfer to the front,

- $\mathbf{A 2}-1.75$ s: the left leg single support phase begins; the right leg starts its transfer to the front,

- $\mathbf{A 3}-2.70$ s: the double support phase starts,

- B1 - 6.75 s: the right leg single support phase begins for the step made to the back; the left leg starts its transfer to the back,

- B2 - 7.90 s: the left leg single support phase starts; the right leg starts its transfer to the back,

- B3 -8.70 s: the double support phase begins.

The initial posture is shown in Fig. 7(a). The right ankle is located at $(0.291,-0.330,0.0064)(\mathrm{m})$, the left ankle is at $(0.101,-0.315,0.065)(\mathrm{m})$, the projection of the CoM is at 

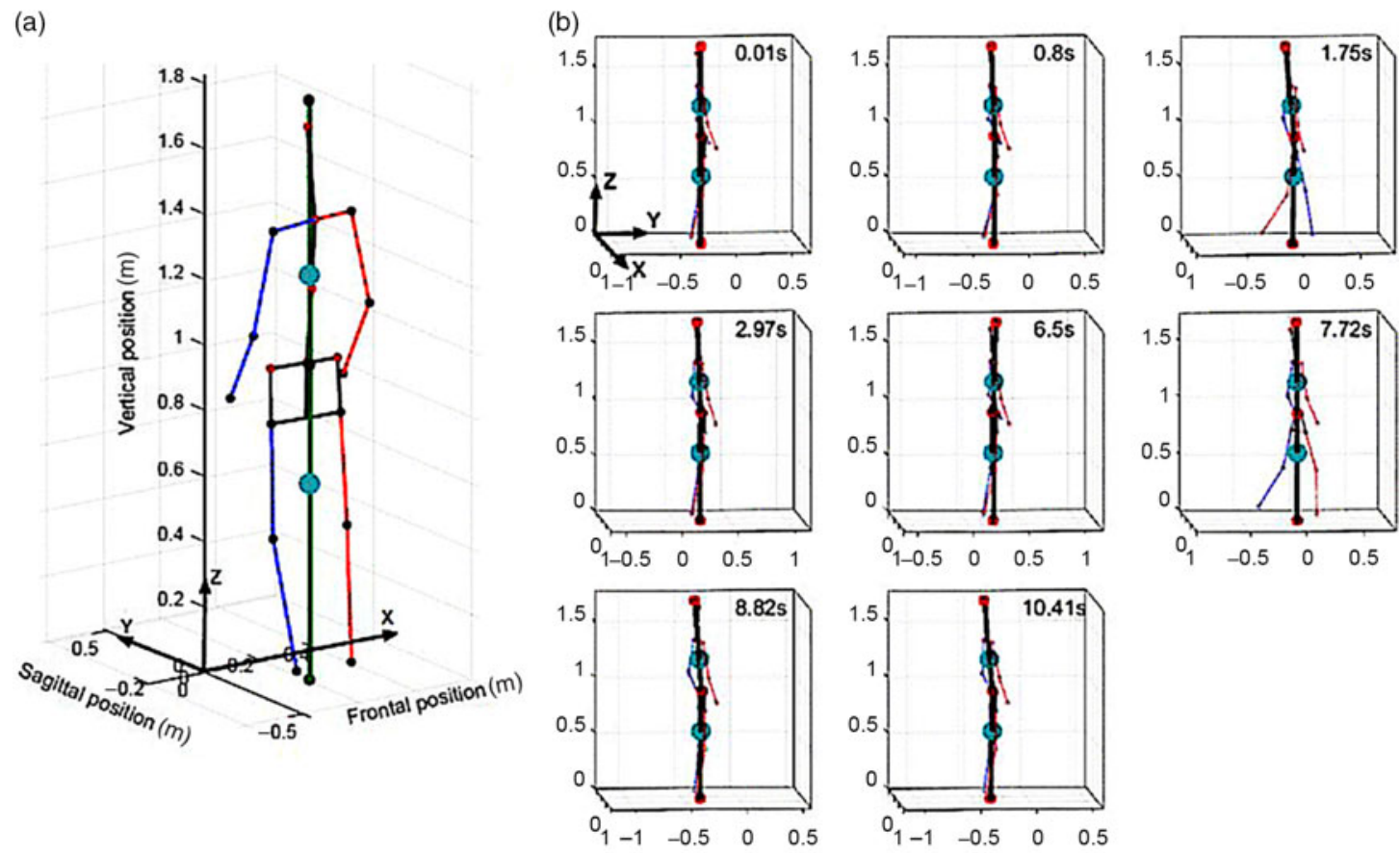

Fig. 7. Making a step forward and back: (a) initial posture, (b) motion frames - visualization of the VDIPC and the stick diagrams.

$(0.205,-0.239,0.00)(\mathrm{m})$. Figure $7(\mathrm{~b})$ shows the sequence of frames from the motion visualization software developed by us to present the human posture and its VDIPC representation for forward-backward stepping. The motion trajectories of the human and of the relevant pendulum points are shown in Fig. 8.

It is easy to notice (Fig. 8(a)) that the wave crests are associated with the gait phases (bounded by the gait events: A1, A2, B1, and B2). The crests for the sideways motion (along the $X$ axis) correspond to the single supports (of each foot) which confirms that the body inclines to the side of the supporting leg. This phenomenon is also observed in a regular walk; therefore, the motion direction cannot be deduced by only using the $X$ trajectories. The small peaks at the beginning (A3) and the end of the double support phase (B1) suggest that the person adjusts his/her stability to assume a more robust position before interchanging the supporting legs.

The motion in the sagittal axis ( $Y$ axis) is shown in Fig. 8(b). Between events A3 and B1 (during the double support phase), the trajectories are almost flat - there is no significant displacement along the $\mathrm{Y}$ axis - the person is standing still. The position increment due to the forward step and decrement when stepping back is clearly visible.

The motion trajectories along the $Z$-axis are illustrated in Fig. 8(c). They show small periodic changes, with the COM position minima appearing during A2 and B2 events, beginnings of single support phases. The trajectories of selected points on the pendulum (joint, tip, and CoM) are offset from the waist trajectory. The upper joint trajectory overlaps the waist trajectory.

Next the ZMP trajectories were obtained for the stick model (with point masses of the segments) of the human body and for the VDIPC. It was investigated how accurately the human ZMP can be reproduced using the VDIPC model. The ZMP position is expressed by (e.g., Kajita et al. ${ }^{19}$ ):

$$
\begin{aligned}
p_{x} & =\frac{\sum_{i}\left[x_{i}\left(m_{i} \ddot{z}_{i}+m_{i} \cdot g\right)\right]-\sum_{i}\left(z_{i} \cdot m_{i} \ddot{x}_{i}\right)}{\sum_{i}\left(m_{i} \ddot{z}_{i}+m_{i} \cdot g\right)} \\
p_{y} & =\frac{\sum_{i}\left[y_{i}\left(m_{i} \ddot{z}_{i}+m_{i} \cdot g\right)\right]-\sum_{i}\left(z_{i} \cdot m_{i} \ddot{y}_{i}\right)}{\sum_{i}\left(m_{i} \ddot{z}_{i}+m_{i} \cdot g\right)}
\end{aligned}
$$

where $\left(x_{i}, y_{i}, z_{i}\right)$ is the $i$-th point mass position in the 3D space, $\left(\ddot{x}_{i}, \ddot{y}_{i}, \ddot{z}_{i}\right)$ is the $i$-th mass acceleration, and $g=-9.81\left[\frac{\mathrm{m}}{\mathrm{s}^{2}}\right]$ is the gravitational acceleration. The total number of segments is equal to $n$, 

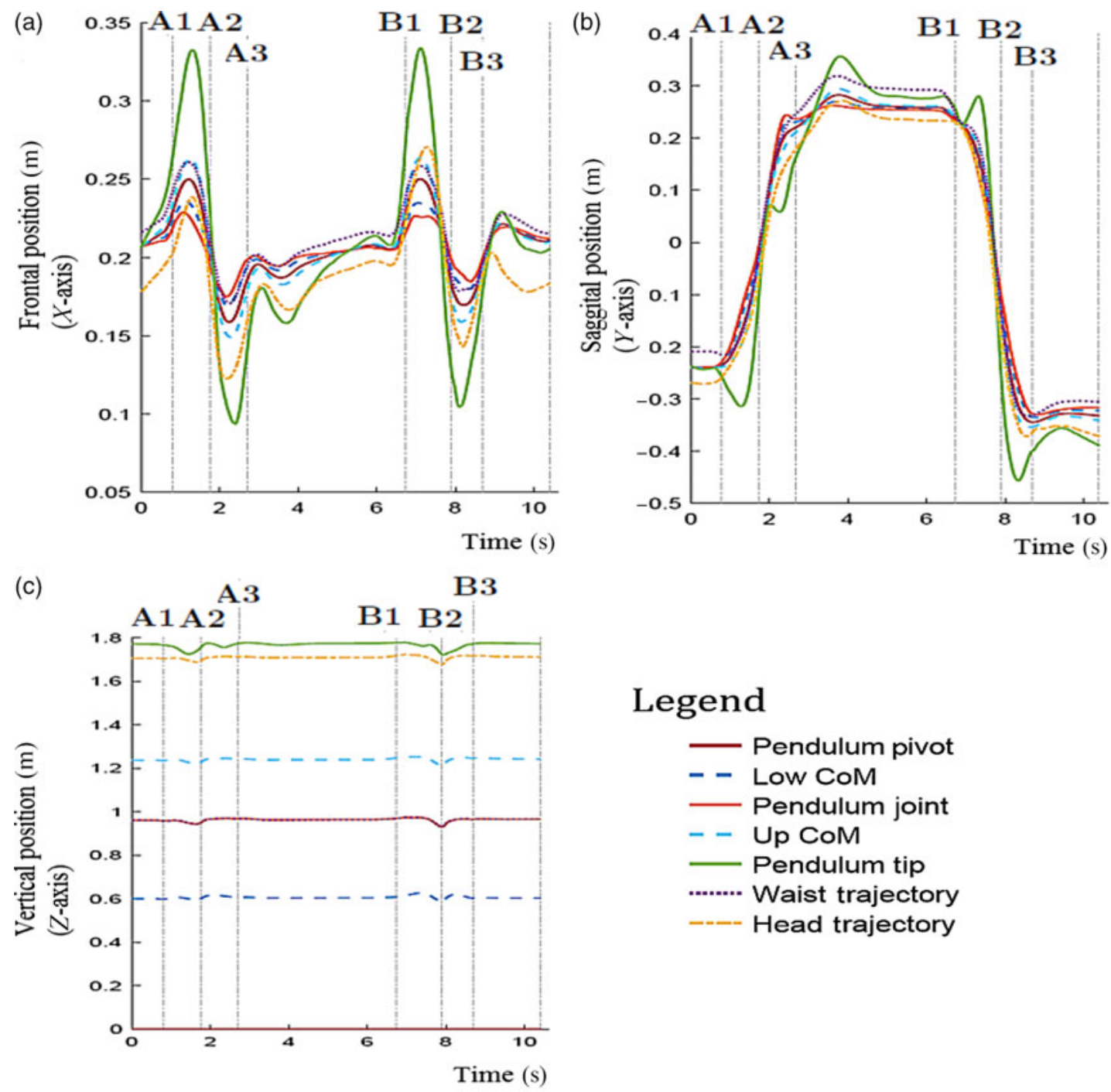

\section{Legend}

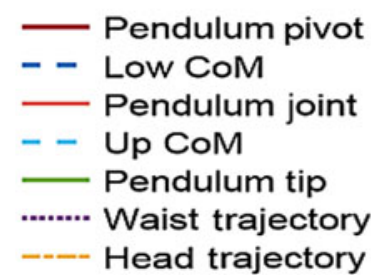

Fig. 8. Making a step forward and back: trajectories of relevant points along: (a) $X$-axis (sideways displacement), (b) $Y$-axis (along the motion direction), (c) $Z$-axis (vertical).

where $n=11$ for the assumed sick diagram of the human body and $n=2$ for the VDIPC model. The accelerations of the point masses were obtained using the numerical form of the position trajectories and computing numerically the second-order derivative.

Figure 9(a) shows the ZMP trajectories for the sick model of the human body and for the VDIPC. The trajectory of the ground projection of the overall CoM is also presented for comparison with the trajectory of the ZMP. The letters marking the motion events are indicated and the footprints are also visualized.

When a person starts the motion, the ZMP overlaps with the CoM projection. The same situation happens when the motion ends. However, the ZMP does not fully coincide with the CoM projection in the single support phases (time periods: A1-A3, B1-B3). The most noticeable discrepancy between $\mathrm{ZMP}$ and CoM projection is for the motion along the $\mathrm{X}$ axis. The ZMP stays inside the support polygon produced by the supporting foot until the support polygon is expanded to a bigger area during the double support phase. Then the ZMP transfers between the supporting feet. In this stage, the ZMP approaches the CoM projection. The ZMP trajectories of the segmented model and of VDIPC almost overlap. This confirms that the VDIPC model defined by us represents well the motion dynamics.

For clarity of presentation, Fig. 9(b) and (c) shows only the trajectories of the projected CoM and ZMP of the VDIPC. Those drawings confirm the mentioned by Vukobratovic et al. ${ }^{36}$ similarity of 
(a)

(a)

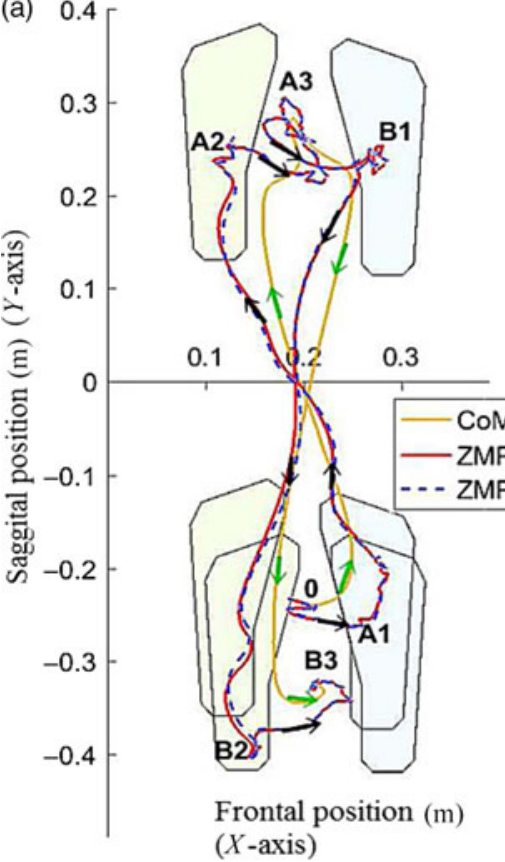

(b)

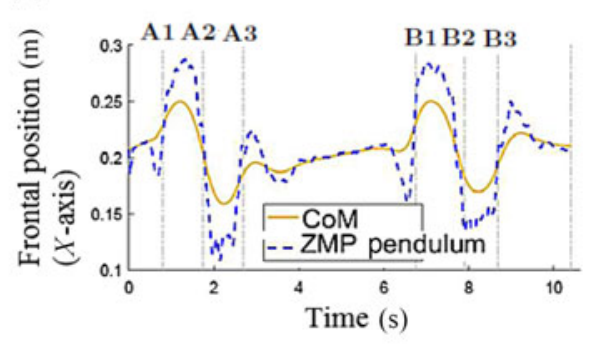

M trajectory

Pegmented model

(c)

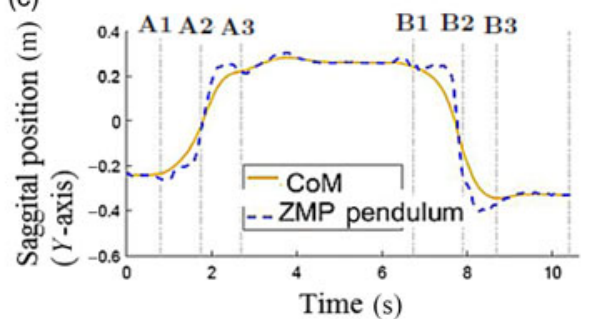

Fig. 9. Making a step forward and back: ZMP and CoM trajectories: (a) support plane view, (b) trajectory along the $X$ axis, and (c) trajectory along the $Y$ axis.

the ZMP position to the CoM projection for quasi-static situations (double support phases - between events $\mathrm{A} 3$ and $\mathrm{B} 1$ ). In general, the CoM position changes in a smaller range than the ZMP, which is especially visible for the $x$ coordinate.

\subsection{Recovery after a frontal push: forced motion}

The person was lightly, but unexpectedly, pushed forward by a sudden impulse to the upper back. The main events of this motion are:

- A1 - 1.02 (s): beginning of the left single support phase, step to the front, from a static double support,

- A2 - 1.94 (s): beginning of the right single support phase, step to the front,

- $\mathbf{A 3}-2.80$ (s): attaining static double support,

- B1 - 6.00 (s): being pushed at the left shoulder,

- B2 -6.50 (s): reaction to the push by placing the right foot to the front to gain stability,

- C1 -7.00 (s): beginning of the posture recovery by stepping back - right single support,

- C2 - 8.53 (s): start of the left single support,

- C3-9.80 (s): attaining the static double support and keeping the position.

The trajectories are shown in Fig. 10. In comparison with the stepping example, a higher asymmetry of shape is visible. For the motion along the $X$-axis (to the side), the sideways inclination to the left in the first left support phase (A1-A2) is smaller than the similar inclination in the second left support phase when stepping back (C2-C3). The $Y$-trajectory depicts the stepping history and the $Z$-trajectory indicates slight decrease of the height for upper link tip and joint at the beginnings of the left single support phase (A2, C2 events).

Figure 11(a) shows the ZMP trajectories for the stick model of a human body and for the VDIPC. The ground projection trajectory of the overall CoM is also presented. The ZMP trajectories indicate that dynamic balancing takes place from the moment of push (B1), because the ZMP moves outside the support polygon. This means that the impulse force (and also torque) is not equilibrated. The person loses balance and gains stability by stepping forward. Following this a backward step is the voluntary return to the previous position. With the recovered postural stability, the course of the ZMP trajectory is similar to that observed in the previous case. 
(a)

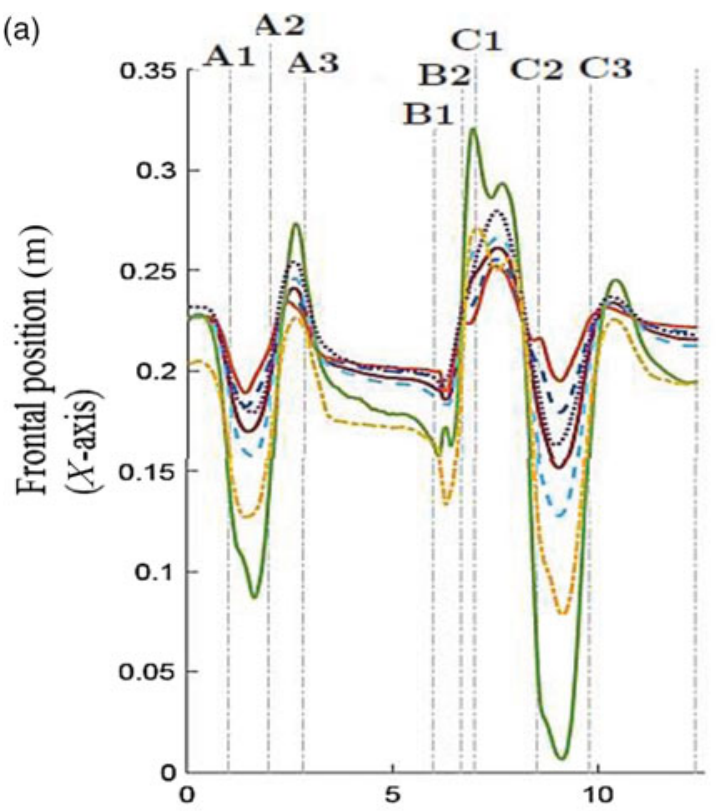

(c) (b)

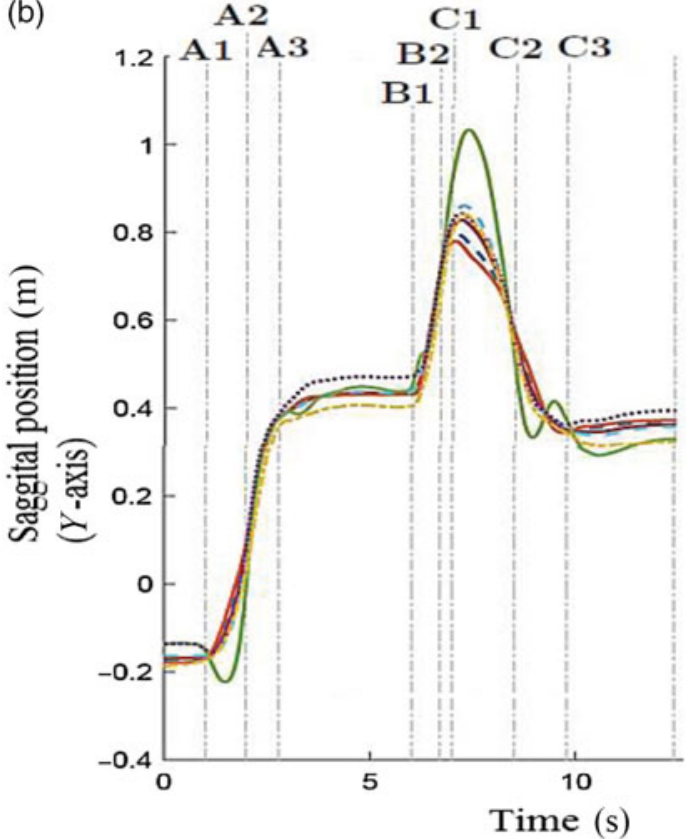

\section{Legend}

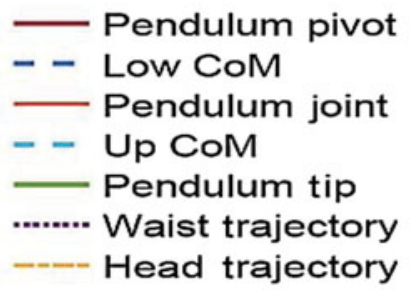


Table I. Average difference between ZMP trajectories for the stick model and VDIPC.

\begin{tabular}{lrr}
\hline Type of movement & \multicolumn{1}{c}{$\overline{\boldsymbol{e}}_{\boldsymbol{x}}(\mathbf{m})$} & \multicolumn{1}{c}{$\overline{\boldsymbol{e}}_{\boldsymbol{y}}(\mathbf{m})$} \\
\hline Normal walk, free arms & $-5.881 \times 10^{-5}$ & $-5.625 \times 10^{-4}$ \\
Normal walk, arms tied to the trunk & $2.744 \times 10^{-4}$ & $2.645 \times 10^{-5}$ \\
Step forward and back & $6.022 \times 10^{-6}$ & $2.945 \times 10^{-5}$ \\
Frontal posh, free arms & $-4.659 \times 10^{-6}$ & $-3.981 \times 10^{-5}$ \\
Frontal push, arms tied to the trunk & $-7.62 \times 10^{-6}$ & $-4.60 \times 10^{-6}$ \\
Side push, free arms & $9.208 \times 10^{-5}$ & $8.362 \times 10^{-5}$ \\
Side push, arms tied to the trunk & $1.866 \times 10^{-4}$ & $-3.270 \times 10^{-5}$ \\
\hline
\end{tabular}

(a)

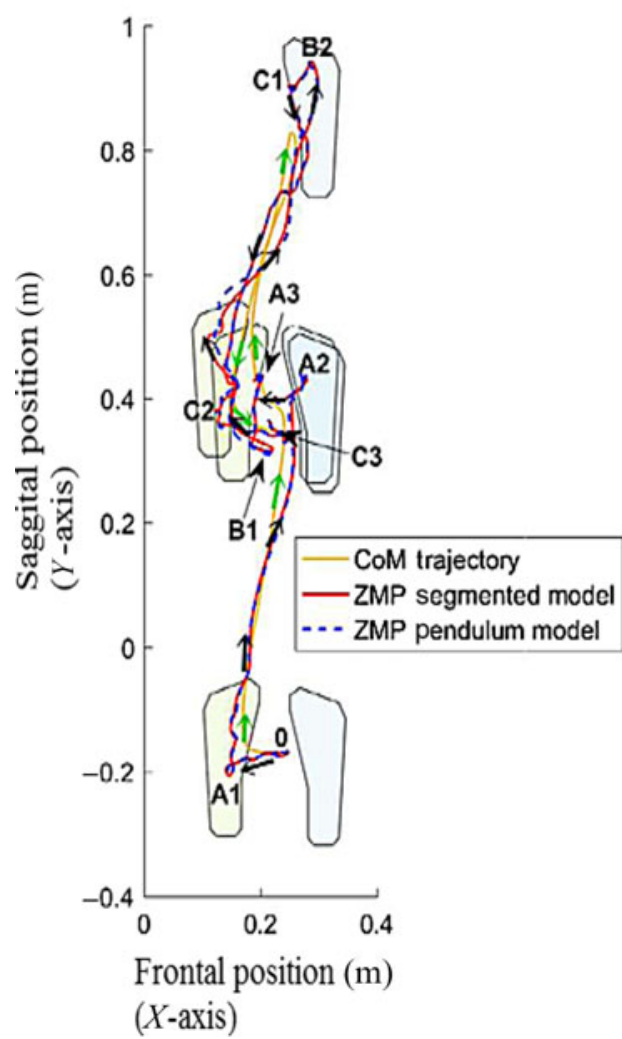

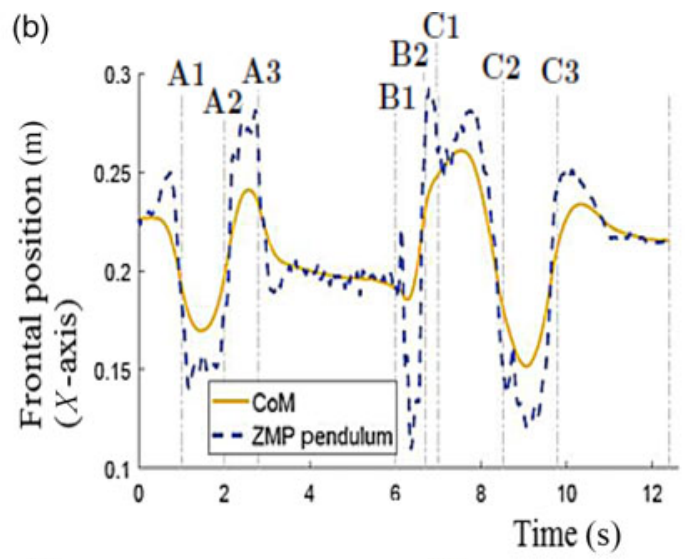

(c)

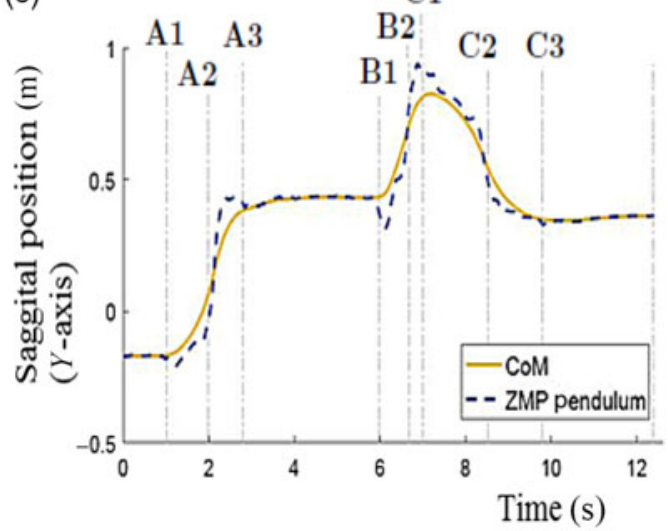

Fig. 11. Push recovery - ZMP and CoM trajectories: (a) support plane view, (b) trajectory along the $X$ axis, and (c) trajectory along the $Y$ axis.

tied arms (b). Motion frames for side push at the upper right shoulder are shown in Fig. 13 for tied arms (a) and free arms (b). The human body model and the reconstructed VDIPC posture are shown. The 3D motion frames were produced by dynamic simulation software specifically elaborated for the presented research. This software enables the comparison of the human and pendulum motion using numerical results and the visual information.

The quantitative evaluation was performed by comparison of the ZMP trajectories of the stick model and the VDIPC models. The results are shown in Table I. The Table contains the average difference between these trajectories obtained along the $X\left(\bar{e}_{x}\right)$ and $Y\left(\bar{e}_{y}\right)$ directions for the set of investigated movements. As it is seen, the absolute differences are not greater than $6 \times 10^{-4}(\mathrm{~m})$; moreover, in all cases the absolute value of maximum difference was less than $0.01(\mathrm{~m})$ along the $X$ direction and less than $0.03(\mathrm{~m})$ along the $Y$ direction. Referring these data to the typical step length, which is about $0.8(\mathrm{~m})$, the foot length $0.25(\mathrm{~m})$, and the step width ranging $0.25(\mathrm{~m})$, the discrepancies are small, which additionally justifies the relevance of proposed pendulum. 
(a)

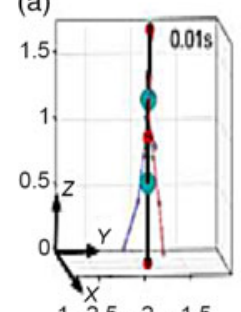

$1-2.5-2-1.5$
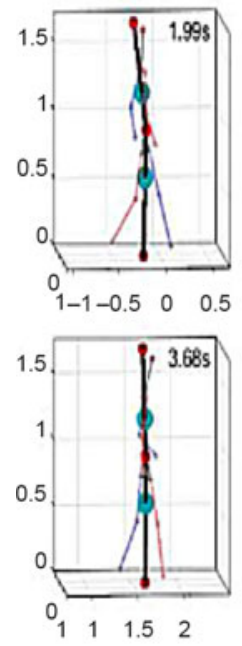
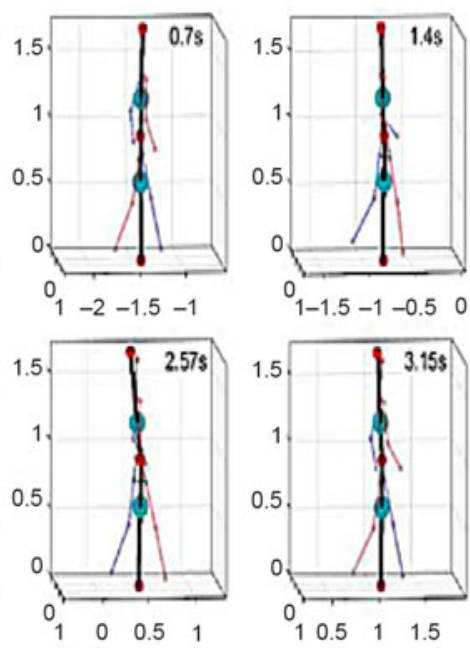

$1-1.5-1-0.50$
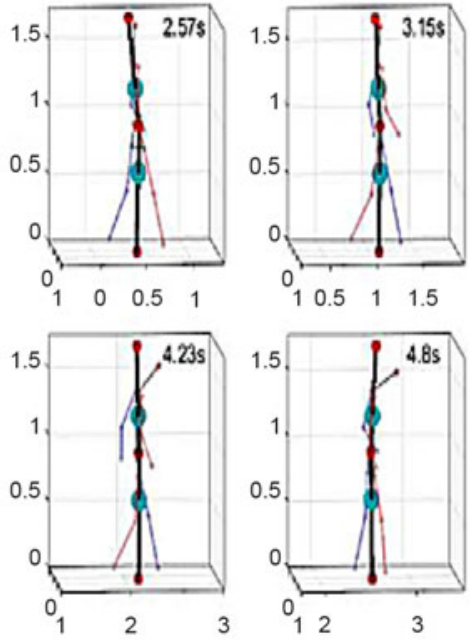

(b)
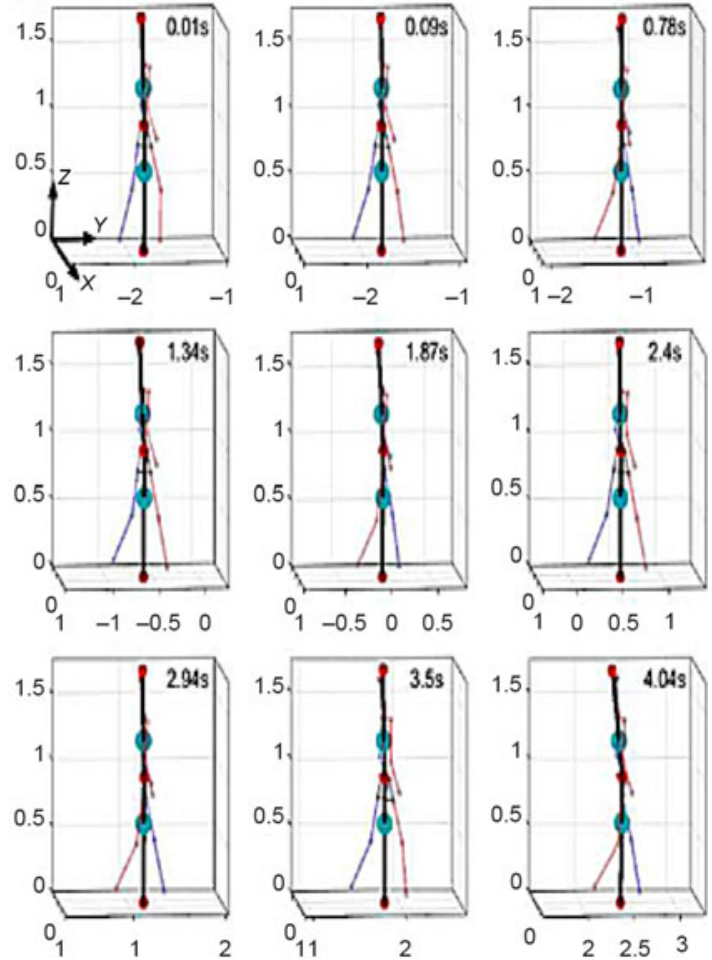

Fig. 12. Sequence of motion frames. Normal walk with free arms (a) and tied arms (b).

(a)
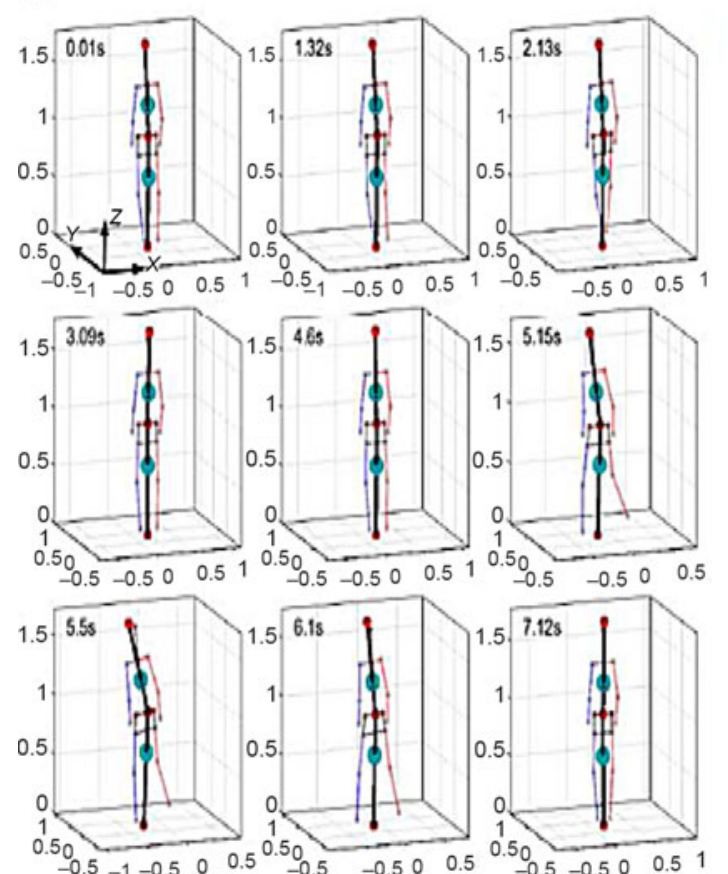

(b)
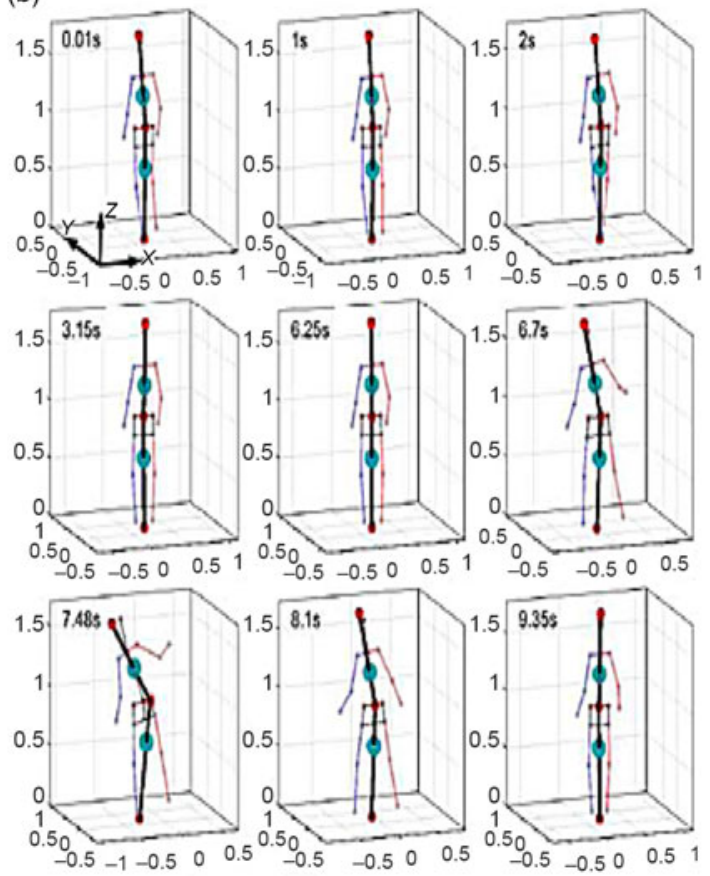

Fig. 13. Sequence of motion frames. Reaction to side push with tied arms (a) and free arms (b). 
(a)

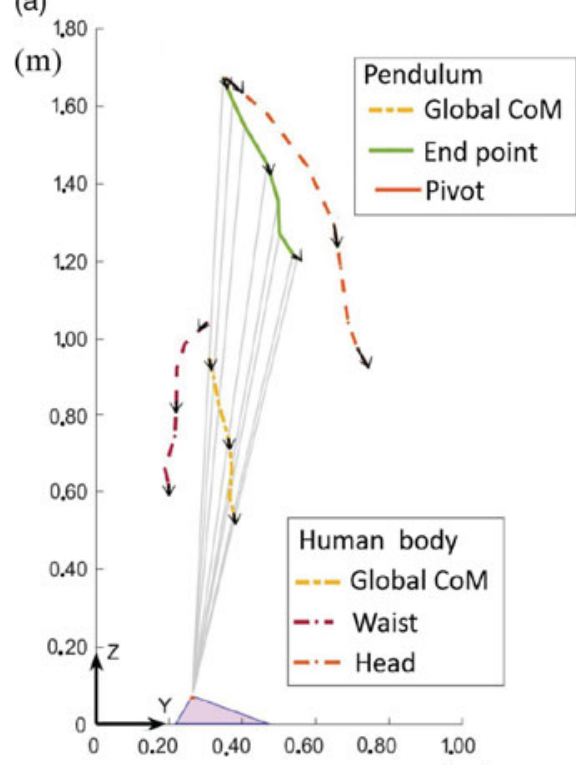

(m) (b)

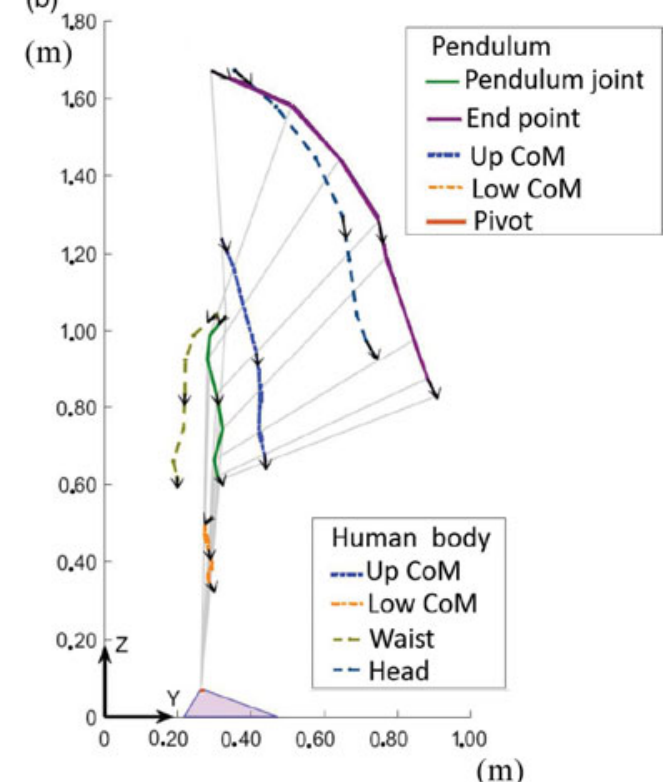

Fig. 14. Single (a) and double (b) inverted pendulum with static pivot point. Not all relevant trajectories of the human body are followed.

\section{Usability of VDIP Models}

\subsection{Comparison of a single and the proposed DIP}

In Villalobos, ${ }^{34}$ it was shown that a single inverted pendulum is not sufficient for representing the ZMP trajectories even for simple forward and side stepping with upright posture, but the classic DIP suffices in this case. To justify the research presented in this paper, the behavior of a single inverted pendulum and a DIP was additionally compared for different postures. The comparison confirmed the inadequacy of a single inverted pendulum. For illustration, let us consider the situation presented in Fig. 4. Two arrangements were tested. In Fig. 14, the pivot point is static and is located in the ankle joint. Figure 14(a) shows that for a single pendulum the information about the waist position is lost. Moreover, the trajectory of the pendulum tip differs much from the head trajectory. The DIP in Fig. 14(b) follows the position of the lower and upper human body masses. The shape of its middle joint trajectory is closer to the waist trajectory, but still with some error. The trajectory of the tip is nearer to the trajectory of the head than that of a single pendulum. In Fig. 15, the pendulum with moving pivot point is considered. The single inverted pendulum in this case is even worse than before (Fig. 15(a)), but the VDIPC follows well not only the lower and upper mass but also the waist trajectory and the trajectory of the head (Fig. 15(b)). This confirms that a DIP represents the relevant information about the body posture.

\subsection{Formal descriptions}

The complete 3D models of the DIP (stationary or on a cart) are not presented in this work. Such models are widely available in the literature devoted to inverted pendulum controllers (e.g., Niemann et al., ${ }^{25}$ Neusser et al. $^{24}$ ). However to justify the usefulness of the elaborated variable pendulum concept, some details are presented.

Figure 16(a) shows the DIP model. The following notation is used: $m_{1}$ - mass of lower part, $m_{2}$ - mass of upper part, $l_{1 m}^{v}$ - the distance from the pivot point to mass $m_{1}, l_{1}^{v}$ - the length of lower part, $l_{2 m}^{v}$ - the distance from middle joint to mass $m_{2}$, and $l_{2}$-length of the upper part. Superscript $v$ denotes a variable parameter. In Fig. 16(a), the pendulum configuration refers to the sagittal plane, but next parts of this figure illustrate $3 \mathrm{D}$ representation, which will be discussed later. The pendulum angles are $\theta_{1}$ and $\theta_{2}$. The pendulum is located on a massless cart to represent human 
(a)

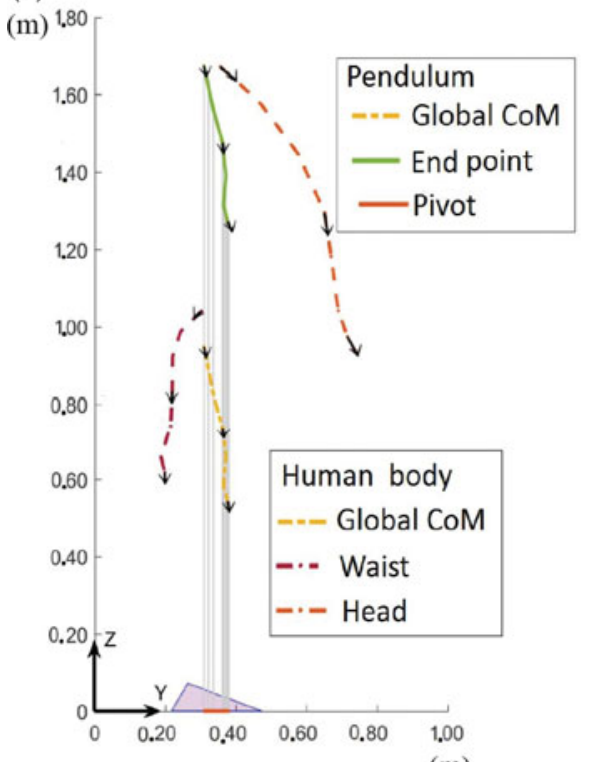

$(\mathrm{m})$ (b)

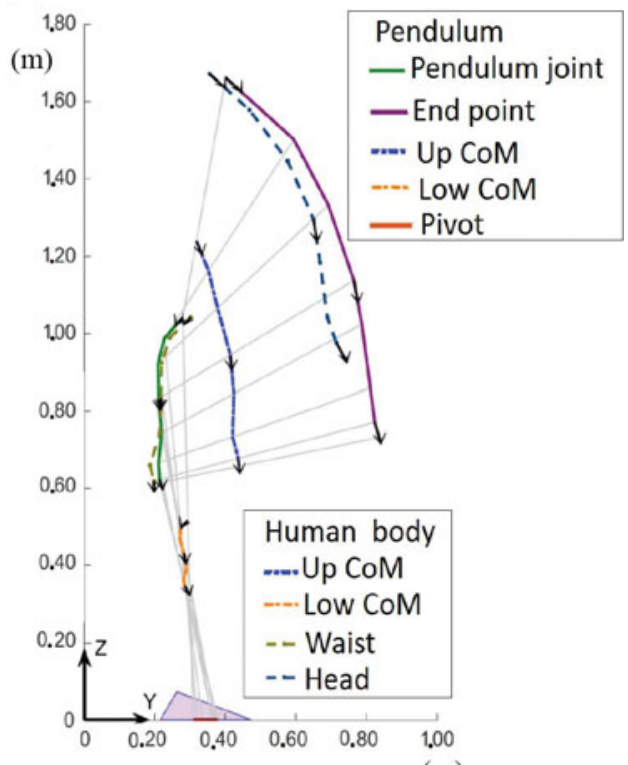

(m)

Fig. 15. Single (a) and double (b) inverted pendulum (VDIPC) with displacing pivot point. The VDIPC follows the relevant trajectories.
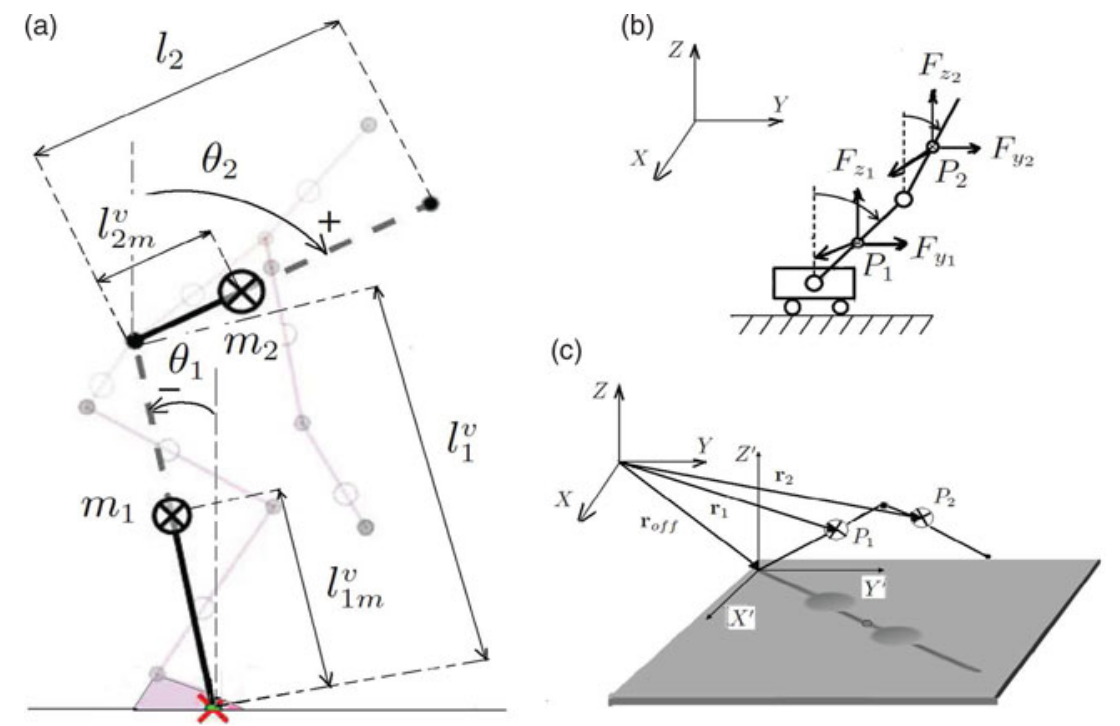

Fig. 16. View of VDIPC and its parameters: (a) planar configuration, (b) forces and reference frames, and (c) general notation.

(humanoid) displacement (Fig. 16(b)). The coordinates of pendulum point masses $m_{1}$ and $m_{2}$ are $P_{1}\left(x_{P_{1}}, y_{P_{1}}, z_{P_{1}}\right)$ and $P_{2}\left(x_{P_{2}}, y_{P_{2}}, z_{P_{2}}\right)$, respectively. The forces acting on point masses are:

$$
\begin{aligned}
& F_{x_{1}}=\ddot{x}_{P_{1}} m_{1}, \quad F_{y_{1}}=\ddot{y}_{P_{1}} m_{1}, \quad F_{z_{1}}=\left(\ddot{z}_{P_{1}}-g\right) m_{1} \\
& F_{x_{2}}=\ddot{x}_{P_{2}} m_{2}, \quad F_{y_{2}}=\ddot{y}_{P_{2}} m_{2}, \quad F_{z_{2}}=\left(\ddot{z}_{P_{2}}-g\right) m_{2}
\end{aligned}
$$

where $g=9.81 \mathrm{~m} / \mathrm{s}$ is the gravitational acceleration.

In humanoids motion synthesis using the preview control method (e.g., Kajita et al. ${ }^{17,19}$ ), the controller produces the ZMP trajectory. In this case, the state variables describe the motion of a single inverted pendulum point mass with respect to the pivot point. The ZMP trajectory is described along the motion direction. This trajectory is transferred to the 2D plane using the planned footprints. 
Finally, the robot motion generation is the inverse problem to the above. The movement of the body parts is obtained considering robot kinematics and using planned ZMP trajectory; the footprints and the state variables describing the point mass motion are treated as constraints. With this set of data, the kinematic redundancy problem is avoided, and the robot posture is obtained without problem. Taking into account the preview control concept, the state equations for the VDIPC are now formulated. The robot moves along the $Y$ axis. The condition for postural equilibrium in the sagittal plane is the starting point of the description. In equilibrium, the torque around the $X$ axis is equal to zero. Following the literature (e.g., Kajita et al. ${ }^{18}$ and Szumowski et al. ${ }^{33}$ ), the vertical accelerations of point masses are neglected together with the acceleration of the pivot point. This leads to the following equilibrium condition:

$$
p_{y}\left(m_{1}+m_{2}\right) g-y_{P_{1}} m_{1} g-y_{P_{2}} m_{2} g-z_{P_{1}} m_{1} \ddot{y}_{P_{1}}-z_{P_{2}} m_{2} \ddot{y}_{P_{2}}=0 .
$$

From the above, the coordinate $p_{y}$ of the ZMP is obtained:

$$
p_{y}=y_{P_{1}} \frac{m_{1}}{m_{1}+m_{2}}+y_{P_{2}} \frac{m_{2}}{m_{1}+m_{2}}+z_{P_{1}} \frac{m_{1}}{\left(m_{1}+m_{2}\right) g} \ddot{y}_{P_{1}}+z_{P_{2}} \frac{m_{2}}{\left(m_{1}+m_{2}\right) g} \ddot{y}_{P_{1}} \text {. }
$$

Using (6), the state and output equations are formulated. The positions of VDIPC point mass together with the derivatives $\left(x_{1}=y_{P_{1}}, . ., x_{4}=y_{P_{1}}, ..\right)$ are selected as state variables. The state equation is as follows:

$$
\left[\begin{array}{l}
\dot{x}_{1} \\
\dot{x}_{2} \\
\dot{x}_{3} \\
\dot{x}_{4} \\
\dot{x}_{5} \\
\dot{x}_{6}
\end{array}\right]=\left[\begin{array}{llllll}
0 & 1 & 0 & 0 & 0 & 0 \\
0 & 0 & 1 & 0 & 0 & 0 \\
0 & 0 & 0 & 0 & 0 & 0 \\
0 & 0 & 0 & 1 & 0 & 0 \\
0 & 0 & 0 & 0 & 1 & 0 \\
0 & 0 & 0 & 0 & 0 & 0
\end{array}\right]\left[\begin{array}{l}
x_{1} \\
x_{2} \\
x_{3} \\
x_{4} \\
x_{5} \\
x_{6}
\end{array}\right]+\left[\begin{array}{ll}
0 & 0 \\
0 & 0 \\
1 & 0 \\
0 & 0 \\
0 & 0 \\
0 & 1
\end{array}\right]\left[\begin{array}{l}
u_{1} \\
u_{2}
\end{array}\right]
$$

The output equation obtained for (6) and (7) produces the ZMP trajectory $\left(y=p_{y}\right)$ :

$$
y=\left[\frac{m_{1}}{m_{1}+m_{2}}, 0, \quad z_{P_{1}} \frac{m_{1}}{\left(m_{1}+m_{2}\right) g}, \frac{m_{2}}{m_{1}+m_{2}}, 0, \quad z_{P_{2}} \frac{m_{2}}{\left(m_{1}+m_{2}\right) g}\right]\left[\begin{array}{l}
x_{1} \\
x_{2} \\
x_{3} \\
x_{4} \\
x_{5} \\
x_{6}
\end{array}\right]
$$

The control signals $u_{1}=\dddot{y}_{P_{1}}, u_{2}=\dddot{y}_{P_{3}}$ are produced by the preview controller. They create the inputs to the robot model described by (7) and (8). Controller synthesis for the formulated above VDIPC model is a serious research problem by itself and is beyond the scope of this paper.

Now the general equilibrium criterion for the VDIPC will be formulated. The criterion states that all torques produced by the force vectors $\mathbf{F}_{\mathbf{i}}$ acting on the system are equilibrated by the inertial and external moments. The equilibrium is formulated with respect to some selected point, which further on is referred to as an equilibrium point. The following notation is used (see Fig. 16(c)): $\mathbf{r}_{\text {off } f}$ the offset vector between the origin of the reference frame and the point with respect to which the torque equilibrium condition is formulated; $\mathbf{I}_{m}$ is the inertia matrix with respect to the $X^{\prime}, Y^{\prime}, Z^{\prime}$ axes of the reference frame which is shifted by $\mathbf{r}_{\text {off }}$ with respect to the absolute reference frame $X, Y, Z ; \omega$ is the rotation velocity vector around the $X^{\prime}, Y^{\prime}, Z^{\prime}$ axes; $\mathbf{M}_{E}$ is the moment from external sources with respect to the equilibrium point; $\mathbf{r}_{i}$ is the vector from the origin of the reference frame to the point $i$ where the force $\mathbf{F}_{i}$ is applied. In the case of double pendulum, equilibrium condition takes the form:

$$
\left(\mathbf{r}_{1}-\mathbf{r}_{o f f}\right) \times \mathbf{F}_{1}+\left(\mathbf{r}_{2}-\mathbf{r}_{o f f}\right) \times \mathbf{F}_{2}=\mathbf{I}_{m} \dot{\omega}+\omega \times \mathbf{I}_{m} \omega+\mathbf{M}_{E} .
$$

This formula enables the study of the conditions for postural equilibrium in 3D space taking into account the VDIPC model. 
Lets us consider the simple planar case which can be used for investigating the postural balance when standing $\mathbf{r}_{\text {off }}=0$. The aim is to maintain the posture; therefore, the pivot point does not move. The coordinates of point masses are:

$$
\begin{aligned}
& x_{P_{1}}=0, \quad y_{P_{1}}=l_{1 m}^{v} \sin \left(\theta_{1}\right), \quad z_{P_{1}}=l_{1 m}^{v} \cos \left(\theta_{1}\right), \\
& x_{P_{2}}=0, \quad y_{P_{2}}=l_{1}^{v} \sin \left(\theta_{1}\right)+l_{2 m}^{v} \sin \left(\theta_{2}\right), \quad z_{P_{2}}=l_{1}^{v} \cos \left(\theta_{1}\right)+l_{2 m}^{v} \cos \left(\theta_{2}\right) .
\end{aligned}
$$

By using (10), the accelerations of point masses are evaluated by double differentiation. Based on (5), the forces acting on point masses are expressed as:

$$
\begin{aligned}
& F_{x_{1}}=0, \quad F_{y_{1}}=\left[l_{1 m}^{v} \cos \left(\theta_{1}\right) \ddot{\theta}_{1}-l_{1 m}^{v} \sin \left(\theta_{1}\right) \dot{\theta}_{1}^{2}\right] m_{1}, \\
& F_{z_{1}}=\left[-l_{1 m}^{v} \sin \left(\theta_{1}\right) \ddot{\theta}_{1}-l_{1 m}^{v} \cos \left(\theta_{1}\right) \dot{\theta}_{1}^{2}-g\right] m_{1}, \\
& F_{x_{2}}=0, \quad F_{y_{2}}=\left[-l_{1}^{v} \sin \left(\theta_{1}\right) \dot{\theta}_{1}^{2}+l_{1}^{v} \cos \left(\theta_{1}\right) \ddot{\theta}_{1}+l_{2 m}^{v} \cos \left(\theta_{2}\right) \ddot{\theta}_{2}-l_{2 m}^{v} \sin \left(\theta_{2}\right) \dot{\theta}_{2}^{2}\right] m_{2}, \\
& F_{z_{2}}=\left[-l_{1}^{v} \cos \left(\theta_{1}\right) \dot{\theta}_{1}^{2}-l_{1}^{v} \sin \left(\theta_{1}\right) \ddot{\theta}_{1}-l_{2 m}^{v} \sin \left(\theta_{2}\right) \ddot{\theta}_{2}-l_{2 m}^{v} \cos \left(\theta_{2}\right) \dot{\theta}_{2}^{2}-g\right] m_{2} .
\end{aligned}
$$

Using (9) and taking into account that the rotation angles are measured counterclockwise, the equilibrium condition around the $X$ axis assumes the form:

$$
y_{P_{1}} F_{z_{1}}+y_{P_{2}} F_{z_{2}}-z_{P_{1}} F_{y_{1}}-z_{P_{2}} F_{y_{2}}=-I_{m_{1}} \ddot{\theta}_{1}-I_{m_{2}}\left(\ddot{\theta}_{1}+\ddot{\theta}_{2}\right)+M_{E} .
$$

where $I_{m_{1}}$ is the inertia of the first link and $I_{m_{2}}$ is the inertia of the second link. Note that in this case the scalar $M_{E}$ is the external torque only with respect to the $X$ axis.

In the upright posture $\cos \left(\theta_{i}\right) \sim 1$ and $\sin \left(\theta_{i}\right) \sim 0$, therefore (10) and (12) can be simplified, and after substituting into (12) the simplified versions of (10) and (12), the torques equilibrium condition assumes the form:

$$
\left(l_{1 m}^{v}\right)^{2} \ddot{\theta}_{1} m_{1}+\left(l_{1}^{v}+l_{2 m}^{v}\right)\left(l_{1}^{v} \ddot{\theta}_{1}+l_{2 m}^{v} \ddot{\theta}_{2}\right) m_{2}=-I_{m_{1}} \ddot{\theta}_{1}-I_{m_{2}}\left(\ddot{\theta}_{1}+\ddot{\theta}_{2}\right)+M_{E} .
$$

which leads to

$$
\left[\left(l_{1 m}^{v}\right)^{2} m_{1}+\left(l_{1}^{v}+l_{2 m}^{v}\right) l_{1}^{v} m_{2}+I_{m_{1}}+I_{m_{2}}\right] \ddot{\theta}_{1}+\left[\left(l_{1}^{v}+l_{2 m}^{v}\right) l_{2 m}^{v} m_{2}+I_{m_{2}}\right] \ddot{\theta}_{2}=M_{E} .
$$

This equation indicates that equilibrium can be obtained by fast dynamics which is achieved by modifying angular accelerations $\ddot{\theta}_{1}$ and $\ddot{\theta}_{2}$ and by slower changes of posture which results in the modification of variable parameters $l_{1}^{v}, l_{1 m}^{v}, l_{2 m}^{v}$. It is obvious that the subject of postural balance can be further investigated. The expanded postural equilibrium studies taking into account the pendulum variability and wide range of postures are again a serious and broad research topic.

\subsection{Discussion}

The efficiency of the models utilizing an inverted pendulum was verified experimentally by many real-time applications, for example, Chengju Liu et al., ${ }^{5}$ Sugihara et al., ${ }^{32}$ and Szumowski et al. ${ }^{33}$ The dynamical equations of DIP are often solved in real-time for the purpose of fast physical pendulum stabilization, for example, Pathompong Jaiwat et al. ${ }^{28}$ Control of humanoids using inverted pendulum models is not as demanding, because the human body sway is much slower than that of a pendulum. The real-time motion control algorithms using inverted pendulum models leave a lot of space for supplementary computations, if they are needed. In previous sections, the VDIP and VDIPC were described. Their ability to reflect a wider range of postures and motion situations in comparison to those considered by the models that have been available up till now was studied. It was illustrated how well the static postures are represented and how well the introduced model (VDIPC) reflects the motion dynamics. The precision is assessed by inspecting the ZMP trajectories (how close is the ZMP trajectory produced by the proposed inverted pendulum model to the ZMP generated by the human body model) and by comparing the postures of the inverted pendulum and those of the human body. For the purpose of modeling human-like movements and postures, there does not exist an accuracy measure. The human movements are not fully repetitive. Even in a rhythmic gait the discrepancies in 
joint trajectories can reach several degrees. Therefore, the validation of the proposed models should be rather qualitative and not quantitative. However, qualitative judgment in this case is not easy as there are no adequate descriptors. We compared ZMP trajectories, because the ZMP criterion is commonly used as the postural stability indicator for humanoid motion synthesis. Moreover, during the synthesis of non-typical motions the human ZMP is often used as the reference to be followed, but obviously it is not accurately imitated. Taking into account all that has been mentioned above, the validation method used here is considered as sufficient, by the community working on humanoid motion synthesis. The formal considerations presented in this section illustrate how the proposed concepts can be used. The examples indicate some farther research directions.

\section{Conclusions}

Anthropomorphic robots, helping human beings and acting in the environment arranged for humans, should assume postures similar to those of humans. They have done it autonomously. The objective of this paper was to find a suitable simplified model, which provides sufficient information about the posture and motion of the human body, so that they can be reflected in humanoids. In the presented studies, the attention was concentrated on the broad range of postures, including those with bent legs. Such postures have been not investigated extensively and thus have not been described appropriately in the literature. Nevertheless, such motions are of importance to human assisting robots. Based on static and dynamic studies, a novel concept of a pendulum with variable parameters (VDIPC) was proposed, which is the main contribution of this paper. It has been proved that different postures assumed by a human can be described by VDPIC configurations, and variable length of the pendulum lower link is essential for accurate representation of a wide range of postures, as many of them are attained by bending legs. The length of the upper link should be constant, because the human spine does not change shape significantly. The results confirmed that VDIPC describes properly not only different postures but also the dynamic aspects of human motion and provides a reasonable approximation of the human body ZMP.

The presented approach adapts the formal models of classic DIP by introducing variable parameters. The models of DIP are computationally the simplest out of the range of human body models applied to humanoids motion synthesis. Such models are used for designing the robots postural stability control as well. Therefore, VDPIC is useful not only for humanoids motion synthesis but also for synthesis of postural stability control with disturbance rejection when trying to sustain not only the upright postures. The latter case is relevant for robots physically interacting with humans.

Using the VDPIC, the robot posture can be recovered by several methods. One is to build the pendulum state equations and apply preview control approach as it has been outlined in this paper. Here instead of approximating the human body by single mass, as it is done by the single pendulum models, the dynamics of two masses is considered, thus enabling easier recovery of the whole body configurations in comparison to the single mass models.

Another possibility is currently explored by us - teaching by demonstration. The observed complex postures of the human during dynamic actions are represented by VDPIC. Such VDPIC configuration is transformed into the configuration of the VDPIC representing the robot, taking into account its masses and sizes in proportion to the human body. Such VDPIC and the introduced additional task descriptors (e.g., position of the hands) are used for recovering the robot posture. It is an option with respect to the computationally demanding approaches where the robot is expected to imitate rather accurately the human postures (teaching by imitation). In our studies, fulfilling the robot task by fast adjustments of the posture in a human like, but not obviously identical way, is the primary goal.

\section{Acknowledgments}

The postural stability study using VDPIC was done by Gabriel R. Rivera Coba at Warsaw University of Technology (WUT). Results were discussed within WUT and Tianjin University of Technology, School of Mechanical Engineering cooperation conducted within the joint Robotic Research Laboratory. The work on teaching by demonstration using VDPIC is funded by POB Research Centre for Artificial Intelligence and Robotics of Warsaw University of Technology within the Excellence Initiative Program - Research University (ID-UB). 


\section{References}

1. A. V. Alexandrov, V. Lippi, T. Mergner, A. A. Frolov, G. Hettich and D. Husek, "Human-inspired eigenmovement concept provides coupling-free sensorimotor control in humanoid robot," Front. Neurorobot. 11, 1-13 (2017), Article 22.

2. H. Audren, J. Vaillant, A. Kheddar, A. Escande, K. Kaneko and E. Yoshida, "Model Preview Control in Multi-contact Motion-application to a Humanoid Robot," IEEE International Conference on Intelligent Robots and Systems (2014) pp. 4030-4035.

3. A. Asker, S. Assal, M. Ding, J. Takamatsu, T. Ogasawara and M. Mohamed, "Experimental Validation of a Motion Generation Model for Natural Robotics-Based Sit to Stand Assistance and Rehabilitation," ROBIO International Conference on Robotics and Biomimetics (2016) pp. 214-219.

4. D. Berenson, S. Srinivasa and J. Kuffner, "Task Space Regions: A framework for pose-constrained manipulation planning," Int. J. Robot. Res. 12(30), 1435-1460 (2011).

5. C. Liu, J. Ning and Q. Chen, "Dynamic walking control of humanoid robots combining linear inverted pendulum mode with parameter optimization," Int. J. Adv. Robot. Syst. 15(1), 1-15 (2018)

6. R. G. Coba, Analysing of Postural Stabilisation Using Simplified Models of Humanoids Thesis (Warsaw University of Technology, 2018).

7. R. Contini, "Body segment parameters. Part II", Artificial Limbs. Rev. Curr. Dev. 16(1), 1-19 (1972).

8. R. J. Full and D. E. Koditschek, "Templates and anchors: Neuromechanical hypotheses of legged locomotion on land," J. Exp. Biol. 202(23), 3325-3332 (1999).

9. C. C. Gordon, T. Churchill, C. E. Clauser, B. Bradtmiller and J. T. McConville, Anthropometric Survey of US Army Personnel: Methods and Summary Statistics. Technical Report (Anthropology Research Project Inc, Yellow Springs, OH, 1989)

10. K. Harada, E. Yoshida and K. Yokoi, Motion Planning for Humanoid Robots (Springer Science \& Business Media, Springer-Verlag London, 2010).

11. K. Hase and N. Yamazaki, "Computer simulation study of human locomotion with a three-dimensional entire-body neuro-musculo-skeletal model”, JSME Int. J. Ser. C Mech. Syst. Mach. Elements Manuf. 45(4), 1040-1050 (2002).

12. Ch. Hayot, S. Sakka, V. Fohanno and P. Lacouture, "Biomechanical modeling of the 3D center of mass trajectory during walking," Movement and Sport Sciences - Science and Motricité. ACAPS, EDP Sciences, pp. 1-11 (2013).

13. K. Hirai, M. Hirose, Y. Haikawa and T. Takenaka, "The Development of Honda Humanoid Robot," IEEE International Conference on Robotics and Automation Proceedings, vol. 2 (1998) pp. 1321-1326.

14. J. Hwang, I. H. Suh, G. Park and T. Kwon, "Human Character Balancing Motion Generation Based on a Double Inverted Pendulum Model," Proceedings of 10-th International Conference on Motion in Games (ACM) (2017) pp. 1-11.

15. S. H. Hyon, J. Morimoto and M. Kawato, "From Compliant Balancing to Dynamic Walking on Humanoid Robot: Integration of CNS and CPG," IEEE International Conference on Robotics and Automation (ICRA) (2010) pp. 1084-1085.

16. A. J. Ijspeert, "Central pattern generators for locomotion control in animals and robots: A review," Neural Networks 21(4), 642-653 (2008).

17. S. Kajita, F. Kanehiro, K. Kaneko, K. Yokoi and H. Hirukawa, "The 3d Linear Inverted Pendulum Model: A Simple Modeling for a Biped Walking Pattern Generation," IEEE/RSJ International Conference on Intelligent Robots and Systems, vol. 1 (2001) pp. 239-246.

18. S. Kajita, F. Kanehiro, K. Kaneko, K. Fujiwara, K. Harada, K. Yokoi and H. Hirukawa, "Biped Walking Pattern Generation by Using Preview Control of Zero Moment Point," IEEE International Conference on Robotics and Automation Proceedings (ICRA), vol. 2 (IEEE, 2003) pp. 1620-1626.

19. S. Kajita, H. Hirukawa, K. Harada and K. Yokoi, Introduction to Humanoid Robotics, vol. 101 (Springer, Springer-Verlag London, 2014).

20. M. Kasaei, N. Lau and A. Pereira, "Comparison Study of Well-Known Inverted Pendulum Models for Balance Recovery in Humanoid Robot," MAPiS 2019 - First MAP-i Seminar Proceedings (2019) pp. 1-6.

21. L. Lanari, S. Hutchinson and L. Marchionni, "Boundedness Issues in Planning of Locomotion Trajectories for Biped Robots," 14th IEEE-RAS International Conference on Humanoid Robots (Humanoids) (2014) pp. 951-958.

22. V. Lippi and T. Mergner, "Human-derived disturbance estimation and compensation (dec) method lends itself to a modular sensorimotor control in a humanoid robot," Front. Neurorobot. 11, 1-49 (2017).

23. I. Mordatch, M. De Lasa and A. Hertzmann, "Robust physics-based locomotion using low-dimensional planning," ACM Trans. Graphics (TOG) 29(4), 71 (2010).

24. Z. Neusser and M. Valasek, "Control of the double inverted pendulum on a cart using the natural motion," Acta Polytechnica 53(6), 883-889 (2013).

25. H. Niemann and J. K. Poulsen, "Design and analysis of controllers for a double inverted pendulum," ISA Trans. 44(1), 145-163 (2005).

26. S. Omran, S. Sakka and Y. Aoustin, "Using the generalized inverted pendulum to generate less energyconsuming trajectories for humanoid walking," Arch. Mech. Eng. 63(2), 245-262 (2016).

27. C. Ott, M. A. Roa and G. Hirzinger, "Posture and Balance Control for Biped Robots Based on Contact Force Optimization," Proceedings of IEEE-RAS International Conference on Humanoid Robots (2011) pp. 26-33. 
28. P. Jaiwat and T. Ohtsuka, "Real-Time Swing-up of Double Inverted Pendulum by Nonlinear Model Predictive Control," ADCONP International Symposium on Advanced Control of Industrial Processes (2014) pp. 290-295.

29. Z. Qiu, A. Escande, A. Micaelli and T. Robert, "A Hierarchical Framework for Realizing DynamicallyStable Motions of Humanoid Robot in Obstacle-Cluttered Environments," Proceedings of IEEE-RAS International Conference on Humanoid Robots (2012) pp.867-874.

30. Z. Shi, X. Huang, T. Hu, Q. Tan and Y. Hou, "Weighted augmented Jacobian matrix with variable coefficient method for kinematics mapping of space teleoperation based on human-robot motion similarity," Adv. Space Res. 56(7), 1401-1416 (2016).

31. N. Scianca, M. Cognetti, D. De Simone, L. L. Lanari and G. Oriolo, "Intrinsically Stable MPC for Humanoid Gait Generation," IEEE-RAS 16th International Conference on Humanoid Robots (Humanoids) (2016) pp. 601-606.

32. T. Sugihara, Y. Nakamura and H. Inoue, "Realtime Humanoid Motion Generation through ZMP Manipulation Based on Inverted Pendulum Control," IEEE International Conference on Robotics and Automation, vol. 2 (2002) pp. 1404-1409.

33. M. Szumowski, M. Zurawska and T. Zielinska, "Preview control applied for humanoid robot motion generation," Arch. Control Sci. 59(LXV)(1), 111-132 (2019).

34. J. Villalobos and T. Zielinska, "Study of postural adjustments for humanoidal helpmates," J. Autom. Mobile Robot. Intell. Syst. 11(04), 15-25 (2017).

35. M. Vukobratovic, Legged Locomotion. Robots and Anthropomorphic Mechanisms (Mihailo Pupin Institute, Belgrade, 1975). Also published in: Japanese (Nikkan Shimbun Ltd. Tokyo, 1975), Russian (MIR, Moscow, 1976), Chinese (Beijing, 1983).

36. M. Vukobratovic and B. Borovac, "Zero-moment point thirty five years of its life," Int. J. Humanoid Robot. 1(01), 157-173 (2004)

37. M. Vukobratovi, V. Potkonjak, K. Babković and B. Borovac, "Simulation model of general human and humanoid motion," Multibody Syst. Dyn. 17(1), 71-96 (2007).

38. R. Walters, "Robotics Answers: Japan Out to Lead the Next Industrial Revolution," [Online]. Available: https://journal.accj.or.jp/robotics-answers-japan-out-to-lead-the-next-industrial-revolution/.

39. T. Wang and C. Chevallereau, "A New Control Law for a 3d Biped Robot Based on Regulation of the Zero Moment Point and Joint Path," 10th IEEE-RAS International Conference on Humanoid Robots (Humanoids) (IEEE, 2010), pp. 27-32.

40. D. A. Winter, Biomechanics and Motor Control of Human Movement (John Wiley \& Sons, 2009).

41. F. Xue, X. Chen, J. Liu and D. Nardi, "Real Time Biped Walking Gait Pattern Generator for a Real Robot," Robot Soccer World Cup (Springer, 2011) pp. 210-221.

42. J. Yamaguchi, E. Soga, S. Inoue and A. Takanishi, "Development of a Bipedal Humanoid Robot Control Method of Whole Body Cooperative Dynamic Biped Walking," IEEE International Conference on Robotics and Automation Proceedings, vol. 1 (IEEE, 1999) pp. 368-374.

43. T. Zielinska, "Coupled oscillators utilised as a gait rhythm generators of two legged walking machine," $J$. Biol. Cybern. 4(03), 263-273 (1996).

44. T. Zielinska and A. Chmielniak, "Biologically inspired motion synthesis method of two-legged robot with compliant feet," Robotica 29(07), 1049-1057 (2011).

45. T. Zielinska, Z. Gao, M. Zurawska, Q. Zheng, T. Mergner and V. Lippi, "Postural Balance Using a Disturbance Rejection Method," 11th IEEE Workshop on Robot Motion and Control (RoMoCo) (2017) pp. 23-28.

46. M. Zurawska, M. Szumowski and T. Zielinska, "Reconfigurable double inverted pendulum applied to the modelling of human robot motion', J. Autom. Mobile Robot. Intell. Syst. 11(2), 12-20 (2017). 